\title{
The Baseline Data Sets for Phase II of the Combined Experiment
}

Mark S. Miller, Derek E. Shipley, Teresa S. Young, Michael C. Robinson, and Marvin W. Luttges

University of Colorado, Boulder, Colorado

David A. Simms

National Renewable Energy Laboratory Golden, Colorado

NREL Technical Monitor:

David A. Simms

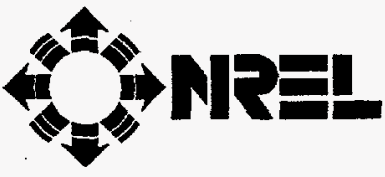

National Renewable Energy Laboratory 1617 Cole Boulevard Golden, Colorado 80401-3393

A national laboratory of the U.S. Department of Energy Managed by Midwest Research Institute for the U.S. Department of Energy under contract No. DE-AC36-83CH10093

Prepared under Subcontract

No. XAO-2-12236-01-103983

July 1995 


\section{NOTICE}

This report was prepared as an account of work sponsored by an agency of the United States government. Neither the United States government nor any agency thereof, nor any of their employees, makes any warranty, express or implied, or assumes any legal liability or responsibility for the accuracy, completeness, or usefulness of any information, apparatus, product, or process disclosed, or represents that its use would not infringe privately owned rights. Reference herein to any specific commercial product, process, or service by trade name, trademark, manufacturer, or otherwise does not necessarily constitute or imply its endorsement, recommendation, or favoring by the United States government or any agency thereof. The views and opinions of authors expressed herein do not necessarily state or reflect those of the United States government or any agency thereof.

Available to DOE and DOE contractors from:

Office of Scientific and Technical Information (OSTI)

P.O. Box 62

Oak Ridge, TN 37831

Prices available by calling (615) $576-8401$

Available to the public from:

National Technical Information Service (NTIS)

U.S. Department of Commerce

5285 Port Royal Road

Springfield, VA 22161

(703) $487-4650$ 


\section{DISCLAIMER}

Portions of this document may be illegible in electronic image products. Images are produced from the best available original document. 


\title{
THE BASELINE DATA SETS FOR PHASE II OF THE COMBINED EXPERIMENT
}

\author{
Mark S. Miller, Derek E. Shipley, Teresa S. Young, Michael C. Robinson, Marvin W. Luttges \\ Department of Aerospace Engineering Sciences \\ University of Colorado, Boulder, CO 80309 \\ and \\ David A. Simms \\ National Renewable Energy Laboratory, Golden, CO 80401
}

\begin{abstract}
The National Renewable Energy Laboratory's "Combined Experiment" was initiated to provide an understanding of horizontal axis wind turbine aerodynamics and their effect on the turbine structure. To this end, aerodynamic and structural baseline data sets have been defined that examine turbine performance under certain conditions. These baseline results have been discussed thoroughly in several papers. This report is an addendum to those papers and supplies additional information about the data selected in creating the baseline data sets. Several appendices are included which contain the tape and cycle numbers of the selected data, along with the average and standard deviation values for the inflow conditions, velocity, and yaw.
\end{abstract}

\section{INTRODUCTION}

Wind turbines operate in a highly unsteady, threedimensional environment that is radically different from the steady-state conditions of a wind tunnel. One of the primary objectives of the "Combined Experiment" is to understand the differences between the wind turbine blade's aerodynamic performance in the field and in steady-state wind tunnel tests. This understanding would significantly benefit wind turbine designers who currently rely upon twodimensional wind tunnel tests to estimate aerodynamic performance.

As an initial step towards this goal, the aerodynamic and the structural performance of a field operating turbine was examined under the steadiest conditions possible. The baseline performance under relatively steady inflow conditions, yaw and velocity, was established for the Combined Experiment turbine and was compared to corresponding wind tunnel data. Off-nominal or anomalous responses of the turbine to unsteady inlet flow conditions could then be carefully identified and quantified.
Previous efforts by the authors focused on establishing aerodynamic and structural performance baselines (Shipley et al., 1994a; Robinson et al., 1994; and Young, 1994). This paper represents an addendum to these reports and presents additional information regarding the particular sequences of data used to establish the performance metrics.

\section{DISCUSSION}

Aerodynamic and structural effects are dependent upon the inflow conditions, velocity and yaw. Previous methods of examining the data included binning by average velocity, and yaw (Huyer, 1992). However, the stochastic distribution of the data suggests that binning and averaging over large portions of the data resulted in poor resolution for both velocity and yaw as well as for aerodynamic events (Young, 1994). Through examination of the wind turbine data, the authors concluded that the best means of analyzing the data was on a individual cycle basis, instead of binning. A cycle is defined as a single rotation of the instrumented blade from $0^{\circ}$ azimuth, the blade straight up, to $360^{\circ}$ azimuth. Therefore, the baseline data sets were established through averaging of single rotational cycles of data or small groups of cycles.

The aerodynamic baseline data set was established using normal and tangent force coefficients. The structural performance was examined using the root flapwise and edgewise bending moments, the low speed shaft torque, and generator power. This particular data was chosen due to the high reliability (Miller et al., 1994a).

The aerodynamic baseline data set is required to be as close to wind tunnel conditions as possible so that the comparison will be accurate. Yaw is the angle between the direction the turbine is facing and the direction the wind is blowing. At yaw angles other than $0^{\circ}$, the wind turbine blade's angle of attack 
variability in inflow conditions, the turbine yaw must be limited to values close to $0^{\circ}$. In addition, the wind turbine aerodynamics were checked for repeatability under similar conditions, as in steady-state wind tunnel tests. It was hypothesized that consistent inflow conditions should provide repeatable aerodynamic results. This hypothesis was examined using two methods. The first used consistent inflow conditions, the three cycle average method, and the second examined consistent aerodynamic data, the correlational method. The data from these two methods was then compared to prove the hypothesis.

These two methods and the results are discussed indepth in Young (1994) and Robinson et al. (1994). The inflow data used for these methods is located in Appendix A and B for the three-cycle-average method and the correlational method, respectively. Figure 1 combines the normal force coefficient, $C_{n}$, data from both methods onto a single graph. The angle of attack values were derived using geometric relationships described in Shipley et al. (1994b).

Appendix A contains the three-cycle-averaged inflow and standard deviation values from the cycles used to create the consistent inflow conditions portion of the aerodynamic baseline data set. The three-cycleaverage method averages together three consecutive cycles with consistent-inflow conditions, velocity, and yaw. The velocity was considered consistent if the standard deviation remained within $\pm 5 \%$ of the mean velocity. The mean yaw values for the cycles included in the baseline were kept between $-1.5^{\circ}$ and $1.5^{\circ}$ and the standard deviation values were maintained below $\pm 2.5^{\circ}$.

Appendix B contains the inflow information from the cycles used to create the repeatable aerodynamic results part of the $0^{\circ}$ yaw baseline data set. The correlational method was used to create this part of the baseline data set. This method used 50 consecutive cycle sets that had the most consistent inflow conditions at $0^{\circ}$ yaw and at velocities of approximately $5,8.5,10,15$, and 20 meters per second $(\mathrm{m} / \mathrm{s})$. The program BESTCYC.F was used to determine which 50 consecutive cycle set were chosen (Miller et al., 1994b). Each cycle in a 50cycle set was correlated to every other cycle in that particular set using Pearson's correlation coefficient. If the correlation coefficient was greater than 0.8 , then the cycles were considered to be highly correlated and, thus, were included in the baseline data set. For more detailed information about the correlational method, refer to Young (1994).
The structural baseline data sets were created to examine aerodynamic effects on turbine structural loads and power generation. In addition, changes in aerodynamics due to operation at yaw angles other than $0^{\circ}$ yaw were studied. Baselines were produced for $-20^{\circ},-10^{\circ}, 10^{\circ}$, and $20^{\circ}$ yaw, and were compared to the $0^{\circ}$ yaw baseline. The cycles used for these baselines were selected in a manner similar to that of the consistent inflow approach used for the $0^{\circ}$ yaw baseline. However, due to the tendency of a downwind horizontal axis wind turbine to align itself with the local flow direction, it was not possible to compile a large enough sample set at yaws other than $0^{\circ}$ using three-cycle groupings. Instead single rotational cycles that exhibited extremely consistent inflow conditions were used. The program CNCTMAX.F, discussed in Miller et al. (1994b), was used to create a list of cycles with the desired cycleaveraged values. The 50 cycles with the most consistent inflow conditions were selected from this list to be the baseline data. The results of the comparison are discussed in Shipley et al. (1994a) and the cycle averaged inflow and standard deviation values are located in Appendix C.

\section{CONCLUSION}

The different baselines created have proven useful in examining the Combined Experiment data. The $0^{\circ}$ yaw baseline has allowed the differences between wind turbine and wind tunnel performance to be studied. These differences have highlighted the importance of unsteady responses in the wind turbine operating under field conditions. In addition, the $0^{\circ}$ yaw baseline proved the author's hypothesis that consistent inflow condition yield repeatable aerodynamic results. The various baselines have also provided insight into the changes in aerodynamics and the effect of aerodynamic response on power generation and turbine structure due to changes in yaw angle.

\section{REFERENCES}

Huyer, S. (1992): Examination of Forced Unsteady Separated Flow Fields on a Rotating Wind Turbine Blade, NREL/TP-442-4864, National Renewable Energy Laboratory, Golden, $\mathrm{CO}$.

Miller, M.S., D.E. Shipley, M.C. Robinson, M.W. Luttges, and D.A. Simms (1994a): Determination of Data Reliability for Phase II of the Combined Experiment, NREL/TP-442-6914, National Renewable Energy Laboratory, Golden, $\mathrm{CO}$. 
Miller, M.S., D.E. Shipley, T.S. Young, M.C. Robinson, M.W. Luttges, and D.A. Simms (1994b): Combined Experiment Phase II Data Characterization, NREL/TP-442-6916, National Renewable Energy Laboratory, Golden, CO.

Robinson, M.C., M.W. Luttges, M.S. Miller, D.E. Shipley, T.S. Young (1994): "Wind Turbine Blade Aerodynamics: The Analysis of Field Test Data", Presented at the 13th ASME/ETCE Wind Energy Symposium, New Orleans, LA, January 23-26, 1994, NREL/TP-441-7108, National Renewable Energy Laboratory, Golden, CO.

Shipley, D.E., M.S. Miller, M.C. Robinson, M.W. Luttges, and D.A. Simms (1994a): "Evidence that Aerodynamic Effects, including Dynamic Stall, Dictate HAWT Structural Loads and Power Generation in Highly Transient Time Frames",
Presented at the Windpower '94 Conference, Minneapolis, MN, May 9-13, 1994, NREL/TP-4417080, National Renewable Energy Laboratory, Golden, CO.

Shipley, D.E., M.S. Miller, M.C. Robinson, M.W. Luttges, and D.A. Simms (1994b): Techniques for the Determination of Local Dynamic Pressure and Angle of Attack on a Horizontal Axis Wind Turbine, NREL/TP-442-7393, National Renewable Energy Laboratory, Golden, CO.

Young, T.S. (1994): "Using Digital Filtering Techniques as an Aid in Wind Turbine Data Analysis", Presented at the AIAA Student Conference, Ft. Collins, CO, April 21-24, 1993, NREL/TP-441-7077, National Renewable Energy Laboratory, Golden, $\mathrm{CO}$.

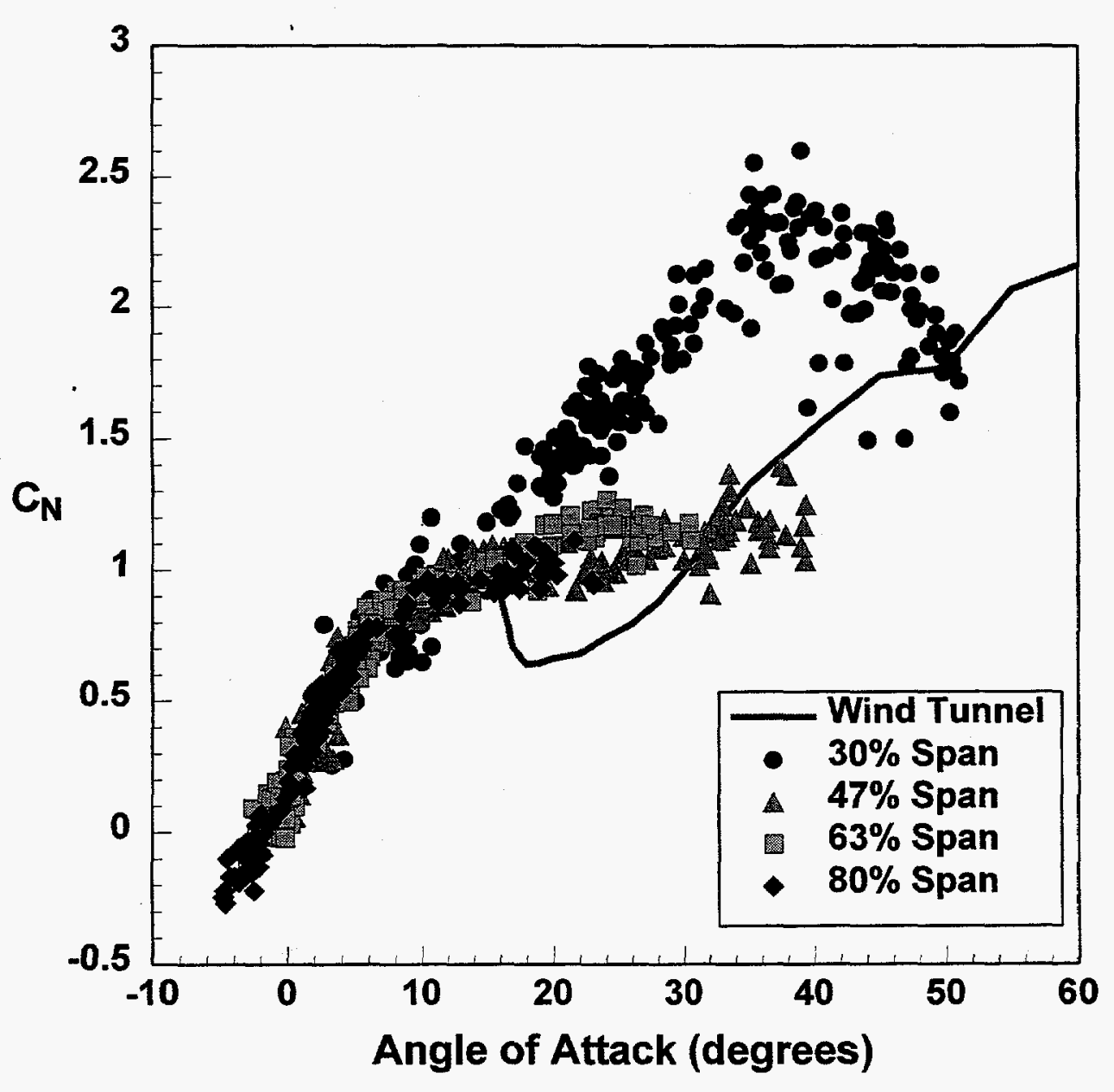

Figure 1. Comparison of independent approaches to wind tunnel data illustrating the differences between wind turbine and wind tunnel aerodynamic performance of normal force coefficient vs. angle-of-attack. 


\section{Appendix A}

This appendix contains the inflow conditions, average, and standard deviation values, for the three cycle average data set. The data in the table was obtained by running the BESTCYC.F program (Miller et al., 1994b). To save time, the program uses the inflow conditions database instead of averaging every single point in the three cycle set. Thus, the average and standard deviation values in the table are occasionally different from those obtained if every single point is averaged together. This difference is very small, usually one hundredth separates the two values.

THREE-CYCLE-AVERAGED INFLOW AND STANDARD DEVIATION VALUES FOR THE CYCLES USED IN THE $0^{\circ}$ YAW BASELINE DATA SET.

Note: The $80 \%$ span data could not be used for cycles with a ${ }^{*}$ next to the tape number due to errors in the data.

\begin{tabular}{|c|c|c|c|c|c|}
\hline Tape & Cycles & Avg. Vel. & Vel. Std. Dev. & Avg. Yaw & Yaw Std. Dev. \\
\hline $\mathrm{d} 066022$ & 294 to 296 & 8.94 & 0.19 & -0.30 & 0.53 \\
\hline $\mathrm{d} 066031$ & 235 to 237 & 12.78 & 0.12 & -0.17 & 1.56 \\
\hline $\mathrm{d} 067011$ & 75 to 77 & 10.91 & 0.14 & 0.06 & 0.59 \\
\hline $\mathrm{d} 067011$ & 76 to 78 & 10.90 & $\overline{0.16}$ & 0.18 & 0.71 \\
\hline $\mathrm{d} 067011$ & 166 to 168 & 11.03 & 0.31 & 0.86 & 1.13 \\
\hline $\mathrm{d} 067011$ & 167 to 169 & 10.74 & 0.25 & -0.31 & 0.54 \\
\hline d067011 & 223 to 225 & 8.87 & 0.13 & 0.34 & 0.18 \\
\hline $\mathrm{d} 067011$ & 224 to 226 & 8.75 & 0.18 & 0.65 & 0.34 \\
\hline $\mathrm{d} 067011$ & 225 to 227 & 8.53 & 0.15 & 0.66 & 0.33 \\
\hline $\mathrm{d} 067012$ & 110 to 112 & 10.11 & 0.13 & -0.18 & 0.54 \\
\hline $\mathrm{d} 067012$ & 111 to 113 & 9.94 & 0.14 & -0.02 & 0.36 \\
\hline $\mathrm{d} 067012$ & 112 to 114 & 9.83 & 0.09 & -0.55 & 0.40 \\
\hline $\mathrm{d} 067012$. & 113 to 115 & 9.84 & 0.09 & -0.15 & 0.86 \\
\hline $\mathrm{d} 067021$ & 326 to 328 & 6.43 & 0.03 & -0.38 & 0.44 \\
\hline $\mathrm{d} 067031$ & 209 to 211 & 11.45 & 0.10 & -0.75 & 1.10 \\
\hline $\mathrm{d} 068011$ & 56 to 58 & 18.18 & 0.22 & 1.13 & 1.02 \\
\hline $\mathrm{d} 068011$ & 120 to 122 & 14.87 & 0.39 & -0.02 & 1.37 \\
\hline $\mathrm{d} 068012$ & 151 to 153 & 16.85 & 0.73 & -0.44 & 1.16 \\
\hline d068021 & 120 to 122 & 18.14 & 0.21 & 0.81 & 0.51 \\
\hline $\mathrm{d} 068022$ & 66 to 68 & 13.29 & 0.08 & 0.62 & 1.05 \\
\hline $\mathrm{d} 069022$ & 183 to 185 & 10.13 & 0.12 & 0.43 & $\overline{0.26}$ \\
\hline $\mathrm{d} 070011$ & 150 to 152 & 8.60 & 0.09 & 0.51 & 0.59 \\
\hline $\mathrm{d} 070011$ & 152 to 154 & 8.49 & 0.19 & 0.15 & 0.94 \\
\hline $\mathrm{d} 070012$ & 32 to 34 & 7.16 & 0.05 & -0.01 & 0.42 \\
\hline $\mathrm{d} 070022$ & 240 to 242 & 8.23 & $\overline{0.12}$ & -0.48 & 1.07 \\
\hline $\mathrm{d} 070031$ & 171 to 173 & 5.82 & 0.02 & -0.10 & 0.35 \\
\hline $\mathrm{d} 070032$ & 220 to 222 & 6.89 & 0.04 & -0.50 & 1.08 \\
\hline $\mathrm{d} 070032$ & 231 to 233 & 6.41 & 0.11 & 0.22 & 1.01 \\
\hline $\mathrm{d} 071012$ & 291 to 293 & 11.87 & 0.13 & -0.13 & 1.63 \\
\hline $\mathrm{d} 071021$ & 229 to 231 & 17.60 & 0.21 & 0.03 & 0.99 \\
\hline $\mathrm{d} 071021$ & 230 to 232 & 17.76 & 0.03 & -0.49 & 1.55 \\
\hline $\mathrm{d} 071021$ & 258 to 260 & 19.30 & 0.32 & -0.73 & 0.29 \\
\hline $\mathrm{d} 071021$ & 306 to 308 & 18.98 & 0.62 & -0.40 & 0.80 \\
\hline
\end{tabular}


Tape

Cycles

Avg. Vel.

Vel. Std. Dev.

Avg. Yaw

Yaw Std. Dev.

\begin{tabular}{|c|c|c|c|c|c|}
\hline $\mathrm{d} 071032$ & 51 to 53 & 18.52 & 0.15 & 0.22 & 2.02 \\
\hline $\mathrm{d} 071032$ & 52 to 54 & 18.53 & $\overline{0.16}$ & -1.26 & 0.08 \\
\hline $\mathrm{d} 071032$ & 53 to 55 & 18.83 & 0.32 & 0.02 & 1.88 \\
\hline d071032 & 110 to 112 & 17.97 & 0.11 & 0.50 & 0.93 \\
\hline d071032 & 111 to 113 & 18.04 & 0.08 & 0.54 & 0.88 \\
\hline $\mathrm{d} 071042$ & 46 to 48 & 20.45 & 0.35 & -1.07 & 1.23 \\
\hline $\mathrm{d} 071042$ & 238 to 240 & 20.80 & 0.25 & -0.74 & 1.04 \\
\hline $\mathrm{d} 072011$ & 27 to 29 & 15.74 & 0.25 & -0.83 & 1.21 \\
\hline $\mathrm{d} 072011$ & 40 to 42 & 13.66 & 0.23 & 0.44 & 1.65 \\
\hline $\mathrm{d} 072012$ & 21 to 23 & 13.30 & 0.34 & -0.98 & 0.25 \\
\hline $\mathrm{d} 072012$ & 22 to 24 & 13.07 & 0.08 & -0.56 & 0.36 \\
\hline $\mathrm{d} 072012$ & 61 to 63 & 14.65 & 0.14 & -0.35 & 0.79 \\
\hline $\mathrm{d} 072012$ & 241 to 243 & 18.53 & 0.32 & -0.70 & 1.67 \\
\hline $\mathrm{d} 072012$ & 276 to 278 & 15.05 & 0.11 & 0.00 & 1.05 \\
\hline d072021 & 10 to 12 & 18.79 & 0.54 & -1.34 & 1.04 \\
\hline d072021 & 11 to 13 & 18.20 & 0.56 & -0.29 & 0.61 \\
\hline $\mathrm{d} 072021$ & 12 to 14 & 17.82 & 0.19 & -0.13 & 0.42 \\
\hline $\mathrm{d} 072042$ & 231 to 233 & 10.24 & 0.16 & -0.42 & 0.65 \\
\hline $\mathrm{d} 073011^{*}$ & 59 to 61 & 16.07 & 0.41 & -1.00 & 1.60 \\
\hline d073011* & 84 to 86 & 13.66 & 0.13 & 0.41 & $\overline{0.56}$ \\
\hline d073011* & 152 to 154 & 16.87 & 0.38 & 0.99 & 0.87 \\
\hline $\mathrm{d} 073012^{*}$ & 18 to 20 & 10.66 & 0.26 & 0.50 & 0.13 \\
\hline d073012* & 243 to 245 & 11.11 & 0.04 & -0.17 & 1.28 \\
\hline d073012* & 244 to 246 & 11.07 & 0.06 & -0.06 & 1.44 \\
\hline $\mathrm{d} 073012^{*}$ & 336 to 338 & 11.57 & 0.40 & 0.73 & 0.75 \\
\hline d073012* & 352 to 354 & 13.52 & 0.09 & -0.61 & 0.32 \\
\hline $\mathrm{d} 073012^{*}$ & 353 to 355 & 13.52 & 0.09 & 0.38 & 1.63 \\
\hline $\mathrm{d} 073012^{*}$ & 354 to 356 & 13.49 & 0.07 & 0.72 & 1.38 \\
\hline $\mathrm{d} 073012^{*}$ & 355 to 357 & 13.74 & 0.39 & 0.84 & 1.29 \\
\hline d073021* & 183 to 185 & 11.90 & 0.16 & 1.09 & $\overline{0.41}$ \\
\hline $\mathrm{d} 073021^{*}$ & 241 to 243 & 10.90 & 0.10 & 0.15 & 0.68 \\
\hline $\mathrm{d} 075011$ & 259 to 261 & 9.76 & 0.13 & -0.28 & 0.42 \\
\hline $\mathrm{d} 075011$ & 260 to 262 & 9.64 & 0.08 & -0.39 & 0.38 \\
\hline $\mathrm{d} 075011$ & 270 to 272 & 9.21 & 0.14 & -0.86 & 0.74 \\
\hline $\mathrm{d} 075011$ & 271 to 273 & 9.28 & 0.06 & -0.62 & 0.48 \\
\hline $\mathrm{d} 075011$ & 272 to 274 & 9.16 & 0.20 & -0.06 & 0.74 \\
\hline d075011 & 345 to 347 & 7.30 & 0.10 & -0.05 & 0.25 \\
\hline $\mathrm{d} 075011$ & 346 to 348 & 7.36 & 0.02 & -0.04 & 0.26 \\
\hline d075011 & 347 to 349 & 7.29 & 0.10 & 0.04 & 0.26 \\
\hline $\mathrm{d} 075012$ & 325 to 327 & 8.20 & 0.13 & -0.18 & 0.17 \\
\hline $\mathrm{d} 075021$ & 155 to 157 & 8.17 & 0.07 & 0.29 & 0.57 \\
\hline $\mathrm{d} 075022$ & 72 to 74 & 5.55 & 0.02 & -0.01 & 0.39 \\
\hline
\end{tabular}




\section{Appendix B}

INFLOW AVERAGE AND STANDARD DEVIATION VALUES FOR THE 50-CONSECUTIVE-CYCLE SETS.

Tape Cycles

Avg. Vel. $\quad$ Vel. Std. Dev.

Avg. Yaw Yaw Std. Dev.

\begin{tabular}{|c|c|c|c|c|c|}
\hline $\mathrm{d} 067022$ & 36 to 85 & 5.13 & 0.57 & 0.24 & 9.06 \\
\hline $\mathrm{d} 075021$ & 5 to 54 & 8.53 & 0.58 & -0.16 & 4.47 \\
\hline $\mathrm{d} 067031$ & 210 to 259 & 10.02 & 0.68 & 0.31 & 3.99 \\
\hline $\mathrm{d} 073011$ & 52 to 101 & 14.50 & 1.61 & -0.14 & 6.66 \\
\hline $\mathrm{d} 071021$ & 307 to 356 & 19.93 & 2.66 & -0.69 & 10.10 \\
\hline
\end{tabular}

CYCLE-AVERAGED INFLOW AND STANDARD DEVIATION VALUES AT 30\% SPAN FOR THE HIGHLY CORRELATED CYCLES $(>0.8)$ FROM THE 50-CYCLE SETS.

\begin{tabular}{|c|c|c|c|c|c|}
\hline Tape & Cycle & Avg. Vel. & Vel. Std. Dev. & Avg. Yaw & Yaw Std. Dev. \\
\hline $\mathrm{d} 067022$ & 36 & 5.45 & 0.08 & 11.45 & 0.30 \\
\hline $\mathrm{d} 067022$ & 37 & 5.80 & 0.10 & 10.63 & 0.50 \\
\hline d067022 & 38 & 5.95 & 0.04 & 11.53 & 0.93 \\
\hline $\mathrm{d} 067022$ & 39 & $\overline{5.78}$ & $\overline{0.04}$ & 12.98 & 0.63 \\
\hline $\mathrm{d} 067022$ & 40 & 5.56 & 0.10 & 11.15 & 3.54 \\
\hline $\mathrm{d} 067022$ & 41 & 5.21 & 0.08 & 4.12 & 0.29 \\
\hline $\mathrm{d} 067022$ & 42 & 4.98 & 0.02 & 2.44 & 1.29 \\
\hline $\mathrm{d} 067022$ & 43 & 4.99 & 0.03 & -3.01 & 3.05 \\
\hline $\mathrm{d} 067022$ & 44 & 5.15 & 0.04 & -8.87 & 0.38 \\
\hline $\mathrm{d} 067022$ & 45 & 5.37 & 0.05 & -7.73 & 2.12 \\
\hline d067022 & 46 & 5.43 & 0.00 & -13.76 & 1.96 \\
\hline $\mathrm{d} 067022$ & 48 & 5.57 & 0.01 & -2.06 & 0.48 \\
\hline $\mathrm{d} 067022$ & 49 & 5.65 & 0.05 & -2.59 & 2.56 \\
\hline $\mathrm{d} 067022$ & 50 & 5.72 & $\overline{0.01}$ & 2.34 & 0.81 \\
\hline d067022 & 51 & 5.77 & 0.05 & 3.61 & 3.06 \\
\hline $\mathrm{d} 067022$ & 52 & 5.91 & $\overline{0.03}$ & -1.58 & 1.55 \\
\hline $\mathrm{d} 067022$ & 53 & 6.02 & $\overline{0.04}$ & 2.20 & 1.73 \\
\hline $\mathrm{d} 067022$ & 54 & 6.10 & 0.02 & 6.64 & 0.93 \\
\hline $\mathrm{d} 067022$ & 55 & 5.94 & 0.07 & 5.30 & 2.02 \\
\hline $\mathrm{d} 067022$ & 56 & 5.75 & 0.04 & 4.99 & 1.40 \\
\hline $\mathrm{d} 067022$ & 57 & 5.67 & 0.06 & 0.26 & 2.58 \\
\hline $\mathrm{d} 067022$ & 58 & 5.33 & $\overline{0.10}$ & 9.28 & 7.00 \\
\hline $\mathrm{d} 067022$ & 59 & 5.05 & 0.05 & 9.35 & 1.77 \\
\hline $\mathrm{d} 067022$ & 60 & 4.97 & 0.02 & 5.47 & 3.16 \\
\hline $\mathrm{d} 067022$ & 61 & 5.15 & $\overline{0.12}$ & 2.28 & 3.56 \\
\hline d067022 & 62 & 5.58 & 0.08 & 4.64 & 4.03 \\
\hline $\mathrm{d} 067022$ & 63 & 5.55 & 0.07 & 3.64 & 4.03 \\
\hline $\mathrm{d} 067022$ & 64 & 5.50 & 0.02 & 11.17 & 2.32 \\
\hline $\mathrm{d} 067022$ & 65 & 5.34 & 0.10 & 14.26 & 1.72 \\
\hline $\mathrm{d} 067022$ & 66 & 4.83 & 0.19 & 19.86 & 1.25 \\
\hline $\mathrm{d} 067022$ & 67 & 4.31 & 0.08 & 15.88 & 1.30 \\
\hline $\mathrm{d} 067022$ & 68 & 4.17 & 0.03 & 5.35 & 4.52 \\
\hline
\end{tabular}


$\begin{array}{llllll}\text { Tape } & \text { Cycle } & \text { Avg. Vel. } & \text { Vel. Std. Dev. } & \text { Avg. Yaw } & \text { Yaw Std. Dev. }\end{array}$

\begin{tabular}{|c|c|c|c|c|c|}
\hline $\mathrm{d} 067022$ & 69 & 4.42 & 0.07 & 5.73 & 2.66 \\
\hline $\mathrm{d} 067022$ & 71 & 4.76 & 0.09 & 1.12 & 1.23 \\
\hline $\mathrm{d} 067022$ & 78 & 4.45 & 0.03 & -10.61 & 2.31 \\
\hline $\mathrm{d} 067022$ & 79 & 4.40 & 0.01 & -7.31 & 1.14 \\
\hline $\mathrm{d} 067022$ & 80 & 4.34 & 0.05 & -13.65 & 2.06 \\
\hline $\mathrm{d} 067022$ & 81 & 4.19 & 0.03 & -10.21 & 2.60 \\
\hline $\mathrm{d} 067022$ & 82 & 4.14 & 0.01 & -7.81 & 2.07 \\
\hline $\mathrm{d} 067022$ & 85 & 4.60 & 0.09 & -12.65 & 1.66 \\
\hline & & & & & \\
\hline $\mathrm{d} 075021$ & 5 & 8.67 & 0.03 & -3.05 & 0.35 \\
\hline $\mathrm{d} 075021$ & 6 & 8.51 & 0.04 & 0.46 & 1.86 \\
\hline $\mathrm{d} 075021$ & 7 & 8.39 & 0.03 & -1.85 & 1.16 \\
\hline d07.5021 & 8 & 8.44 & 0.03 & -2.96 & 1.23 \\
\hline $\mathrm{d} 075021$ & 9 & 8.54 & 0.08 & -2.29 & 1.28 \\
\hline $\mathrm{d} 075021$ & 15 & 8.64 & 0.06 & 6.70 & 0.86 \\
\hline $\mathrm{d} 075021$ & 16 & 8.70 & 0.01 & 2.65 & 0.48 \\
\hline $\mathrm{d} 075021$ & 17 & 8.56 & 0.07 & 4.09 & 1.44 \\
\hline d075021 & 18 & 8.78 & 0.09 & 5.25 & 1.73 \\
\hline $\mathrm{d} 075021$ & 20 & 9.00 & 0.08 & 6.43 & 1.43 \\
\hline d075021 & 21 & 8.90 & 0.05 & 0.77 & 0.80 \\
\hline $\mathrm{d} 075021$ & 22 & 8.89 & 0.04 & 0.93 & 2.38 \\
\hline $\mathrm{d} 075021$ & 23 & 9.19 & 0.12 & -0.02 & 1.40 \\
\hline $\mathrm{d} 075021$ & 24 & 9.55 & 0.10 & 2.64 & 2.31 \\
\hline d075021 & 25 & 9.80 & 0.08 & -3.89 & 1.96 \\
\hline $\mathrm{d} 075021$ & 26 & 9.94 & 0.03 & -5.41 & 3.01 \\
\hline d075021 & 28 & 9.59 & 0.15 & -5.35 & 0.88 \\
\hline $\mathrm{d} 075021$ & 29 & 9.11 & 0.11 & -4.18 & 0.62 \\
\hline $\mathrm{d} 075021$ & 30 & 9.00 & 0.07 & -5.56 & 1.39 \\
\hline d075021 & 31 & 9.17 & 0.03 & -6.67 & 0.91 \\
\hline d075021 & 32 & 8.86 & 0.14 & -6.16 & 0.81 \\
\hline $\mathrm{d} 075021$ & 33 & 8.65 & 0.01 & -4.00 & 0.58 \\
\hline $\mathrm{d} 075021$ & 34 & 8.52 & 0.06 & -5.16 & 1.71 \\
\hline $\mathrm{d} 075021$ & 35 & 8.38 & 0.05 & -6.02 & 0.78 \\
\hline $\mathrm{d} 075021$ & 36 & 8.25 & 0.01 & -7.10 & 0.72 \\
\hline $\mathrm{d} 075021$ & 37 & 8.21 & 0.05 & -8.12 & 0.76 \\
\hline d075021 & 38 & 8.23 & 0.06 & -4.35 & 2.74 \\
\hline d075021 & 39 & 8.47 & 0.04 & -0.69 & 1.11 \\
\hline d075021 & 41 & 8.28 & 0.08 & 1.51 & 0.62 \\
\hline $\mathrm{d} 075021$ & 42 & 8.07 & 0.07 & 3.79 & 1.10 \\
\hline $\mathrm{d} 075021$ & $\overline{43}$ & 7.90 & 0.03 & 2.93 & 0.48 \\
\hline d075021 & 44 & 7.89 & 0.02 & 1.80 & 0.49 \\
\hline $\mathrm{d} 075021$ & 45 & 7.93 & 0.05 & 2.10 & 0.57 \\
\hline $\mathrm{d} 075021$ & 46 & 8.15 & 0.03 & 0.01 & 1.45 \\
\hline $\mathrm{d} 075021$ & 47 & 8.11 & 0.04 & -0.12 & 0.44 \\
\hline d075021 & 48 & 8.10 & 0.05 & 4.49 & 2.04 \\
\hline $\mathrm{d} 075021$ & 49 & 8.16 & 0.01 & 0.76 & 2.41 \\
\hline d075021 & 50 & 8.08 & 0.03 & 0.47 & 0.43 \\
\hline
\end{tabular}


Tape

Cycle

Avg. Vel.

Vel. Std. Dev.

Avg. Yaw Yaw Std. Dev.

\begin{tabular}{|c|c|c|c|c|c|}
\hline $\mathrm{d} 075021$ & 51 & 8.16 & 0.01 & 1.29 & 1.03 \\
\hline $\mathrm{d} 075021$ & 52 & 7.97 & 0.11 & 0.78 & 2.56 \\
\hline $\mathrm{d} 075021$ & 53 & 7.61 & 0.09 & 0.75 & 2.51 \\
\hline $\mathrm{d} 075021$ & 54 & 7.46 & 0.03 & -3.01 & 1.89 \\
\hline & & & & & \\
\hline $\mathrm{d} 067031$ & 213 & 11.24 & 0.10 & -0.75 & 3.34 \\
\hline $\mathrm{d} 067031$ & 223 & 10.57 & 0.04 & -1.29 & 1.32 \\
\hline $\mathrm{d} 067031$ & 224 & 10.37 & 0.14 & -0.19 & 1.12 \\
\hline $\mathrm{d} 067031$ & 226 & 9.45 & 0.17 & 9.35 & 3.77 \\
\hline d067031 & 228 & 10.04 & 0.06 & -0.04 & 1.66 \\
\hline $\mathrm{d} 067031$ & 229 & 9.67 & 0.24 & -0.01 & 0.82 \\
\hline $\mathrm{d} 067031$ & 230 & 9.25 & 0.03 & -2.38 & 2.08 \\
\hline $\mathrm{d} 067031$ & 231 & $\overline{9.36}$ & 0.02 & 0.75 & 3.57 \\
\hline $\mathrm{d} 067031$ & 233 & 9.45 & 0.02 & 5.10 & 1.88 \\
\hline $\mathrm{d067031}$ & 234 & 9.53 & 0.02 & 6.11 & 4.64 \\
\hline $\mathrm{d} 067031$ & 235 & 9.61 & 0.07 & 9.65 & 3.07 \\
\hline d067031 & 236 & 9.98 & 0.13 & 1.06 & 1.21 \\
\hline $\mathrm{d} 067031$ & 237 & 10.26 & 0.06 & 1.52 & 0.78 \\
\hline $\mathrm{d} 067031$ & 243 & 10.24 & 0.02 & 2.40 & 0.53 \\
\hline $\mathrm{d} 067031$ & 247 & 9.99 & 0.11 & 3.03 & 2.79 \\
\hline & & & & & \\
\hline $\mathrm{d} 073011$ & 55 & 16.95 & 0.31 & 5.12 & 0.71 \\
\hline $\mathrm{d} 073011$ & 65 & 16.13 & 0.06 & 6.87 & 1.23 \\
\hline $\mathrm{d} 073011$ & 66 & 15.71 & 0.24 & 10.62 & 4.33 \\
\hline $\mathrm{d} 073011$ & 70 & 15.00 & 0.19 & 4.14 & 1.36 \\
\hline $\mathrm{d} 073011$ & 74 & 14.20 & 0.09 & 0.48 & 0.84 \\
\hline d073011 & 77 & 15.49 & 0.20 & -5.03 & 1.93 \\
\hline $\mathrm{d} 073011$ & 78 & 15.02 & 0.09 & -0.43 & 1.97 \\
\hline $\mathrm{d} 073011$ & 80 & 14.33 & 0.16 & 2.68 & 0.61 \\
\hline $\mathrm{d} 073011$ & 83 & 14.08 & 0.32 & 1.34 & 3.73 \\
\hline $\mathrm{d} 073011$ & 85 & 13.84 & 0.07 & 0.73 & 0.73 \\
\hline $\mathrm{d} 073011$ & 87 & 13.42 & 0.07 & -8.95 & 1.55 \\
\hline d073011 & 88 & 13.05 & 0.15 & -13.99 & 3.83 \\
\hline $\mathrm{d} 073011$ & 90 & 12.50 & 0.49 & -13.92 & 2.80 \\
\hline $\mathrm{d} 073011$ & $\overline{93}$ & 11.56 & 0.07 & -8.43 & 4.87 \\
\hline $\mathrm{d} 073011$ & 95 & 11.72 & 0.26 & 5.71 & 2.81 \\
\hline $\mathrm{d} 073011$ & 97 & 12.82 & 0.14 & 0.93 & 1.63 \\
\hline $\mathrm{d} 073011$ & 99 & 13.31 & 0.03 & -5.63 & 1.61 \\
\hline $\mathrm{d} 071021$ & 308 & 18.64 & 0.05 & 0.51 & 2.53 \\
\hline $\mathrm{d} 071021$ & 309 & 18.25 & 0.33 & -2.45 & 1.68 \\
\hline $\mathrm{d} 071021$ & 310 & 17.55 & 0.09 & 2.86 & 2.11 \\
\hline $\mathrm{d} 071021$ & 311 & 17.93 & 0.11 & 10.05 & 1.24 \\
\hline $\mathrm{d} 071021$ & 312 & 17.25 & 0.16 & 5.87 & 3.17 \\
\hline d071021 & 313 & 16.45 & 0.35 & 0.16 & 5.29 \\
\hline $\mathrm{d} 071021$ & 314 & 15.14 & 0.25 & -7.93 & 0.98 \\
\hline $\mathrm{d} 071021$ & 315 & 14.52 & 0.15 & -14.39 & 7.36 \\
\hline
\end{tabular}




\begin{tabular}{|c|c|c|c|c|c|}
\hline Tape & Cycle & Avg. Vel. & Vel. Std. Dev. & Avg. Yaw & Yaw Std. Dev. \\
\hline $\mathrm{d} 071021$ & 316 & 15.42 & 0.27 & -22.47 & 2.99 \\
\hline $\mathrm{d} 071021$ & 317 & 15.86 & 0.23 & -32.01 & 4.43 \\
\hline $\mathrm{d} 071021$ & 318 & 14.28 & 0.51 & -13.00 & 3.94 \\
\hline $\mathrm{d} 071021$ & 319 & 14.73 & 0.74 & -1.63 & 6.92 \\
\hline $\mathrm{d} 071021$ & 320 & 15.82 & 0.08 & -2.48 & 5.74 \\
\hline $\mathrm{d} 071021$ & 321 & 16.98 & 0.63 & 6.41 & 4.51 \\
\hline $\mathrm{d} 071021$ & 322 & 18.08 & 0.41 & 13.55 & 4.96 \\
\hline $\mathrm{d} 071021$ & 323 & 20.06 & 1.19 & 22.32 & 3.65 \\
\hline $\mathrm{d} 071021$ & 324 & 23.02 & 0.14 & 13.11 & 4.42 \\
\hline $\mathrm{d} 071021$ & 325 & 21.65 & 1.02 & 8.20 & 1.52 \\
\hline $\mathrm{d} 071021$ & 326 & 18.98 & 0.32 & -2.85 & 6.75 \\
\hline $\mathrm{d} 071021$ & 327 & 18.94 & 0.08 & -16.08 & 1.49 \\
\hline $\mathrm{d} 071021$ & 330 & 19.77 & 0.78 & -6.54 & 11.64 \\
\hline $\mathrm{d} 071021$ & 331 & 21.05 & 0.15 & -2.54 & 2.83 \\
\hline $\mathrm{d} 071021$ & 333 & 23.40 & 0.45 & 2.48 & 1.39 \\
\hline $\mathrm{d} 071021$ & 337 & 20.59 & 0.66 & -0.04 & 3.51 \\
\hline $\mathrm{d} 071021$ & 338 & 19.71 & 0.44 & -1.21 & 3.44 \\
\hline $\mathrm{d} 071021$ & 339 & 19.00 & 0.49 & -9.72 & 3.70 \\
\hline $\mathrm{d} 071021$ & 342 & 20.46 & 0.12 & -5.71 & 4.60 \\
\hline $\mathrm{d} 071021$ & 343 & 21.01 & 0.16 & 0.08 & 3.08 \\
\hline $\mathrm{d} 071021$ & 344 & 21.97 & -0.48 & 5.48 & 0.94 \\
\hline $\mathrm{d} 071021$ & 346 & 22.94 & 0.28 & 7.82 & 1.47 \\
\hline $\mathrm{d} 071021$ & 347 & 23.18 & 0.17 & 4.74 & 1.74 \\
\hline $\mathrm{d} 071021$ & 348 & 23.15 & 0.09 & 6.78 & 0.86 \\
\hline $\mathrm{d} 071021$ & 349 & 22.51 & 0.25 & 7.01 & 1.23 \\
\hline $\mathrm{d} 071021$ & 353 & 19.86 & 0.55 & 1.44 & 1.88 \\
\hline $\mathrm{d} 071021$ & 355 & 20.17 & 0.36 & -1.85 & 4.56 \\
\hline $\mathrm{d} 071021$ & 356 & 20.19 & 0.55 & -0.83 & 3.48 \\
\hline
\end{tabular}

CYCLE-AVERAGED INFLOW AND STANDARD DEVIATION VALUES AT 47\% SPAN FOR THE HIGHLY CORRELATED CYCLES $(>0.8)$ FROM THE 50-CYCLE SETS.

\begin{tabular}{|c|c|c|c|c|c|}
\multicolumn{1}{c}{ Tape } & Cycle & Avg. Vel. & Vel. Std. Dev. & Avg. Yaw & Yaw Std. Dev. \\
\hline $\mathrm{d} 067022$ & 36 & 5.45 & 0.08 & 11.45 & 0.30 \\
\hline $\mathrm{d} 067022$ & 37 & 5.80 & 0.10 & 10.63 & 0.50 \\
\hline $\mathrm{d} 067022$ & 38 & 5.95 & 0.04 & 11.53 & 0.93 \\
\hline $\mathrm{d} 067022$ & 39 & 5.78 & 0.04 & 12.98 & 0.63 \\
\hline $\mathrm{d} 067022$ & 40 & 5.56 & 0.10 & 11.15 & 3.54 \\
\hline $\mathrm{d} 067022$ & 41 & 5.21 & 0.08 & 4.12 & 0.29 \\
\hline $\mathrm{d} 067022$ & 42 & 4.98 & 0.02 & 2.44 & 1.29 \\
\hline $\mathrm{d} 067022$ & 43 & 4.99 & 0.03 & -3.01 & 3.05 \\
\hline $\mathrm{d} 067022$ & 44 & 5.15 & 0.04 & -8.87 & 0.38 \\
\hline $\mathrm{d} 067022$ & 45 & 5.37 & 0.05 & -7.73 & 2.12 \\
\hline $\mathrm{d} 067022$ & 46 & 5.43 & 0.00 & -13.76 & 1.96 \\
\hline $\mathrm{d} 067022$ & 47 & 5.49 & 0.03 & -4.87 & 1.44 \\
\hline $\mathrm{d} 067022$ & 48 & 5.57 & 0.01 & -2.06 & 0.48 \\
\hline $\mathrm{d} 067022$ & 49 & 5.65 & 0.05 & -2.59 & 2.56 \\
\hline
\end{tabular}


Tape

\begin{tabular}{|c|c|c|c|c|c|}
\hline lape & Cycle & Avg. vel. & rel. sta. Dev. & Avg. raw & Iaw sta. DeV. \\
\hline$d 067022$ & 50 & 5.72 & 0.01 & 2.34 & 0.81 \\
\hline $\mathrm{d} 067022$ & 51 & 5.77 & 0.05 & 3.61 & 3.06 \\
\hline $\mathrm{d} 067022$ & 53 & 6.02 & 0.04 & 2.20 & 1.73 \\
\hline $\mathrm{d} 067022$ & 54 & 6.10 & 0.02 & 6.64 & 0.93 \\
\hline $\mathrm{d} 067022$ & 55 & 5.94 & 0.07 & 5.30 & 2.02 \\
\hline $\mathrm{d} 067022$ & 56 & 5.75 & 0.04 & 4.99 & 1.40 \\
\hline $\mathrm{d} 067022$ & 57 & 5.67 & 0.06 & 0.26 & 2.58 \\
\hline $\mathrm{d} 067022$ & 50 & 4.97 & 0.02 & 5.47 & 3.16 \\
\hline $\mathrm{d} 067022$ & 61 & 5.15 & 0.12 & 2.28 & 3.56 \\
\hline $\mathrm{d} 067022$ & 63 & 5.55 & 0.07 & 3.64 & 4.03 \\
\hline $\mathrm{d} 067022$ & 65 & $\overline{5.34}$ & 0.10 & 14.26 & 1.72 \\
\hline $\mathrm{d} 067022$ & 66 & 4.83 & 0.19 & 19.86 & 1.25 \\
\hline $\mathrm{d} 067022$ & 67 & 4.31 & 0.08 & 15.88 & 1.30 \\
\hline $\mathrm{d} 067022$ & 68 & 4.17 & 0.03 & 5.35 & 4.52 \\
\hline $\mathrm{d} 067022$ & 71 & $\overline{4.76}$ & 0.09 & 1.12 & 1.23 \\
\hline $\mathrm{d} 067022$ & 76 & $\overline{4.83}$ & 0.02 & -5.47 & 1.19 \\
\hline $\mathrm{d} 067022$ & 77 & 4.65 & 0.08 & -10.67 & 4.82 \\
\hline d067022 & 78 & 4.45 & 0.03 & -10.61 & 2.31 \\
\hline $\mathrm{d} 067022$ & 79 & 4.40 & 0.01 & -7.31 & 1.14 \\
\hline d067022 & 80 & 4.34 & 0.05 & -13.65 & 2.06 \\
\hline $\mathrm{d} 067022$ & 81 & 4.19 & 0.03 & -10.21 & 2.60 \\
\hline $\mathrm{d} 067022$ & 82 & 4.14 & 0.01 & -7.81 & 2.07 \\
\hline $\mathrm{d} 067022$ & 83 & 4.13 & 0.01 & -12.77 & 0.49 \\
\hline $\mathrm{d} 067022$ & 84 & 4.22 & 0.08 & -13.68 & 1.42 \\
\hline $\mathrm{d} 067022$ & 85 & 4.60 & 0.09 & -12.65 & 1.66 \\
\hline d075021 & 5 & 8.67 & 0.03 & -3.05 & 0.35 \\
\hline $\mathrm{d} 075021$ & $\frac{6}{6}$ & 8.51 & 0.04 & 0.46 & 1.86 \\
\hline $\mathrm{d} 075021$ & 7 & 8.39 & 0.03 & -1.85 & 1.16 \\
\hline $\mathrm{d} 075021$ & 8 & 8.44 & 0.03 & -2.96 & 1.23 \\
\hline $\mathrm{d} 075021$ & 9 & 8.54 & 0.08 & -2.29 & 1.28 \\
\hline $\mathrm{d} 075021$ & 12 & 8.01 & 0.13 & 8.66 & 3.16 \\
\hline $\mathrm{d} 075021$ & 15 & 8.64 & 0.06 & 6.70 & $\overline{0.86}$ \\
\hline $\mathrm{d} 075021$ & 16 & 8.70 & 0.01 & 2.65 & 0.48 \\
\hline $\mathrm{d} 075021$ & 17 & 8.56 & 0.07 & 4.09 & 1.44 \\
\hline $\mathrm{d} 075021$ & 18 & 8.78 & 0.09 & 5.25 & 1.73 \\
\hline $\mathrm{d} 075021$ & 20 & 9.00 & 0.08 & $\overline{6.43}$ & 1.43 \\
\hline $\mathrm{d} 075021$ & 21 & 8.90 & 0.05 & 0.77 & 0.80 \\
\hline $\mathrm{d} 075021$ & 23 & 9.19 & 0.12 & -0.02 & 1.40 \\
\hline $\mathrm{d} 075021$ & 25 & 9.80 & 0.08 & -3.89 & 1.96 \\
\hline $\mathrm{d} 075021$ & 26 & 9.94 & 0.03 & -5.41 & 3.01 \\
\hline $\mathrm{d} 075021$ & 27 & 9.84 & 0.02 & -5.13 & 2.75 \\
\hline $\mathrm{d} 075021$ & 30 & 9.00 & 0.07 & -5.56 & 1.39 \\
\hline $\mathrm{d} 075021$ & 32 & 8.86 & 0.14 & -6.16 & 0.81 \\
\hline $\mathrm{d} 075021$ & 33 & 8.65 & 0.01 & -4.00 & 0.58 \\
\hline d075021 & 34 & 8.52 & 0.06 & -5.16 & 1.71 \\
\hline $\mathrm{d} 075021$ & 35 & 8.38 & 0.05 & -6.02 & 0.78 \\
\hline
\end{tabular}


Tape

Cycle

Avg. Vel.

Vel. Std. Dev.

Avg. Yaw

Yaw Std. Dev.

\begin{tabular}{|c|c|c|c|c|c|}
\hline $\mathrm{d} 075021$ & 36 & 8.25 & 0.01 & -7.10 & 0.72 \\
\hline d075021 & 37 & 8.21 & 0.05 & -8.12 & 0.76 \\
\hline $\mathrm{d} 075021$ & 38 & 8.23 & 0.06 & -4.35 & 2.74 \\
\hline $\mathrm{d} 075021$ & 39 & 8.47 & 0.04 & -0.69 & 1.11 \\
\hline $\mathrm{d} 075021$ & 40 & 8.39 & 0.02 & 2.78 & 1.35 \\
\hline $\mathrm{d} 075021$ & 41 & 8.28 & 0.08 & 1.51 & 0.62 \\
\hline $\mathrm{d} 075021$ & 42 & 8.07 & 0.07 & 3.79 & 1.10 \\
\hline $\mathrm{d} 075021$ & 43 & 7.90 & 0.03 & 2.93 & 0.48 \\
\hline $\mathrm{d} 075021$ & 44 & 7.89 & 0.02 & 1.80 & 0.49 \\
\hline $\mathrm{d} 075021$ & 45 & 7.93 & 0.05 & 2.10 & 0.57 \\
\hline $\mathrm{d} 075021$ & 46 & 8.15 & 0.03 & 0.01 & 1.45 \\
\hline d075021 & 47 & 8.11 & 0.04 & -0.12 & 0.44 \\
\hline $\mathrm{d} 075021$ & 48 & 8.10 & $\overline{0.05}$ & 4.49 & 2.04 \\
\hline $\mathrm{d} 075021$ & 49 & 8.16 & 0.01 & 0.76 & 2.41 \\
\hline d075021 & 50 & 8.08 & 0.03 & 0.47 & 0.43 \\
\hline $\mathrm{d} 075021$ & 51 & 8.16 & 0.01 & 1.29 & 1.03 \\
\hline $\mathrm{d} 075021$ & 52 & 7.97 & 0.11 & 0.78 & 2.56 \\
\hline $\mathrm{d} 075021$ & 53 & 7.61 & 0.09 & 0.75 & 2.51 \\
\hline $\mathrm{d} 075021$ & 54 & 7.46 & 0.03 & -3.01 & 1.89 \\
\hline$d 067031$ & 211 & 1133 & 0.12 & -143 & 078 \\
\hline $\mathrm{d} 067031$ & 214 & 11.07 & 0.21 & -4.34 & 0.77 \\
\hline d067031 & 215 & 10.57 & 0.06 & -1.44 & 3.91 \\
\hline $\mathrm{d} 067031$ & 216 & 10.53 & 0.09 & 1.32 & 2.15 \\
\hline $\mathrm{d} 067031$ & 221 & 10.18 & 0.12 & -4.10 & 1.74 \\
\hline $\mathrm{d} 067031$ & 229 & 9.67 & 0.24 & -0.01 & 0.82 \\
\hline $\mathrm{d} 067031$ & 231 & 9.36 & 0.02 & 0.75 & 3.57 \\
\hline d067031 & 233 & 9.45 & 0.02 & 5.10 & 1.88 \\
\hline $\mathrm{d} 067031$ & 237 & 10.26 & 0.06 & 1.52 & 0.78 \\
\hline d067031 & 238 & 10.29 & 0.02 & 0.13 & 0.91 \\
\hline $\mathrm{d} 067031$ & 240 & 10.40 & 0.03 & -0.97 & 1.59 \\
\hline d067031 & 243 & 10.24 & 0.02 & 2.40 & 0.53 \\
\hline $\mathrm{d} 067031$ & 245 & 10.62 & 0.05 & 6.00 & 0.83 \\
\hline $\mathrm{d} 067031$ & 247 & 9.99 & 0.11 & 3.03 & 2.79 \\
\hline $\mathrm{d} 067031$ & 248 & 9.86 & 0.05 & 3.42 & 5.19 \\
\hline $\mathrm{d} 067031$ & 250 & 9.44 & 0.05 & -4.53 & 2.55 \\
\hline & & & & & \\
\hline $\mathrm{d} 073011$ & 64 & 16.06 & 0.11 & 5.59 & 1.08 \\
\hline $\mathrm{d} 073011$ & 70 & 15.00 & 0.19 & 4.14 & 1.36 \\
\hline $\mathrm{d} 073011$ & 71 & 14.43 & 0.14 & 7.73 & 2.10 \\
\hline $\mathrm{d} 073011$ & 74 & 14.20 & 0.09 & $\overline{0.48}$ & 0.84 \\
\hline $\mathrm{d} 073011$ & $\overline{83}$ & 14.08 & 0.32 & 1.34 & 3.73 \\
\hline $\mathrm{d} 073011$ & 90 & 12.50 & 0.49 & -13.92 & 2.80 \\
\hline $\mathrm{d} 073011$ & 92 & 12.01 & 0.32 & -17.02 & 2.21 \\
\hline $\mathrm{d} 073011$ & 93 & 11.56 & 0.07 & $-8 . \overline{43}$ & 4.87 \\
\hline $\mathrm{d} 073011$ & 97 & 12.82 & 0.14 & 0.93 & 1.63 \\
\hline
\end{tabular}


Tape

Cycle

Avg. Vel.

Vel. Std. Dev.

Avg. Yaw

Yaw Std. Dev.

\begin{tabular}{|l|l|l|l|l|l|}
\hline $\mathrm{d} 071021$ & 311 & 17.93 & 0.11 & 10.05 & 1.24 \\
\hline $\mathrm{d} 071021$ & 313 & 16.45 & 0.35 & 0.16 & 5.29 \\
\hline $\mathrm{d} 071021$ & 314 & 15.14 & 0.25 & -7.93 & 0.98 \\
\hline $\mathrm{d} 071021$ & 315 & 14.52 & 0.15 & -14.39 & 7.36 \\
\hline $\mathrm{d} 071021$ & 320 & 15.82 & 0.08 & -2.48 & 5.74 \\
\hline $\mathrm{d} 071021$ & 321 & 16.98 & 0.63 & 6.41 & 4.51 \\
\hline $\mathrm{d} 071021$ & 322 & 18.08 & 0.41 & 13.55 & 4.96 \\
\hline $\mathrm{d} 071021$ & 323 & 20.06 & 1.19 & 22.32 & 3.65 \\
\hline $\mathrm{d} 071021$ & 324 & 23.02 & 0.14 & 13.11 & 4.42 \\
\hline $\mathrm{d} 071021$ & 325 & 21.65 & 1.02 & 8.20 & 1.52 \\
\hline $\mathrm{d} 071021$ & 326 & 18.98 & 0.32 & -2.85 & 6.75 \\
\hline $\mathrm{d} 071021$ & 327 & 18.94 & 0.08 & -16.08 & 1.49 \\
\hline $\mathrm{d} 071021$ & 328 & 21.00 & 1.15 & -17.63 & 1.73 \\
\hline $\mathrm{d} 071021$ & 333 & 23.40 & 0.45 & 2.48 & 1.39 \\
\hline $\mathrm{d} 071021$ & 336 & 22.00 & 0.44 & 5.50 & 1.16 \\
\hline $\mathrm{d} 071021$ & 339 & 19.00 & 0.49 & -9.72 & 3.70 \\
\hline $\mathrm{d} 071021$ & 340 & 19.06 & 0.43 & -12.65 & 2.28 \\
\hline $\mathrm{d} 071021$ & 341 & 20.29 & 0.32 & -13.02 & 2.40 \\
\hline $\mathrm{d} 071021$ & 345 & 23.66 & 0.28 & 7.32 & 3.12 \\
\hline $\mathrm{d} 071021$ & 350 & 23.24 & 0.34 & 4.33 & 0.78 \\
\hline $\mathrm{d} 071021$ & 352 & 21.58 & 0.41 & 0.83 & 1.95 \\
\hline $\mathrm{d} 071021$ & 354 & 19.74 & 0.20 & 2.75 & 2.53 \\
\hline
\end{tabular}

CYCLE-AVERAGED INFLOW AND STANDARD DEVIATION VALUES AT 63\% SPAN FOR THE HIGHLY CORRELATED CYCLES $(>0.8)$ FROM THE 50-CYCLE SETS.

\begin{tabular}{|c|c|c|c|c|c|}
\hline Tape & Cycle & Avg. Vel. & Vel. Std. Dev. & Avg. Yaw & Yaw Std. Dev. \\
\hline $\mathrm{d} 067022$ & 36 & 5.45 & 0.08 & 11.45 & 0.30 \\
\hline $\mathrm{d} 067022$ & 37 & 5.80 & 0.10 & 10.63 & 0.50 \\
\hline $\mathrm{d} 067022$ & 38 & 5.95 & 0.04 & 11.53 & 0.93 \\
\hline $\mathrm{d} 067022$ & 39 & 5.78 & 0.04 & 12.98 & 0.63 \\
\hline $\mathrm{d} 067022$ & 40 & 5.56 & 0.10 & 11.15 & 3.54 \\
\hline $\mathrm{d} 067022$ & 41 & 5.21 & 0.08 & 4.12 & 0.29 \\
\hline $\mathrm{d} 067022$ & 42 & 4.98 & 0.02 & 2.44 & 1.29 \\
\hline $\mathrm{d} 067022$ & 43 & 4.99 & 0.03 & -3.01 & 3.05 \\
\hline $\mathrm{d} 067022$ & 44 & 5.15 & 0.04 & -8.87 & 0.38 \\
\hline $\mathrm{d} 067022$ & 45 & 5.37 & 0.05 & -7.73 & 2.12 \\
\hline $\mathrm{d} 067022$ & 47 & 5.49 & 0.03 & -4.87 & 1.44 \\
\hline $\mathrm{d} 067022$ & 48 & 5.57 & 0.01 & -2.06 & 0.48 \\
\hline $\mathrm{d} 067022$ & 50 & 5.72 & 0.01 & 2.34 & 0.81 \\
\hline $\mathrm{d} 067022$ & 53 & 6.02 & 0.04 & 2.20 & 1.73 \\
\hline $\mathrm{d} 067022$ & 54 & 6.10 & 0.02 & 6.64 & 0.93 \\
\hline $\mathrm{d} 067022$ & 55 & 5.94 & 0.07 & 5.30 & 2.02 \\
\hline $\mathrm{d} 067022$ & 56 & 5.75 & 0.04 & 4.99 & 1.40 \\
\hline $\mathrm{d} 067022$ & 57 & 5.67 & 0.06 & 0.26 & 2.58 \\
\hline $\mathrm{d} 067022$ & 60 & 4.97 & 0.02 & 5.47 & 3.16 \\
\hline $\mathrm{d} 067022$ & 63 & 5.55 & 0.07 & 3.64 & 4.03 \\
\hline
\end{tabular}


Tape

\begin{tabular}{|c|c|}
\hline $\mathrm{d} 067022$ & 65 \\
\hline $\mathrm{d} 067022$ & 66 \\
\hline $\mathrm{d} 067022$ & 67 \\
\hline $\mathrm{d} 067022$ & 75 \\
\hline $\mathrm{d} 067022$ & 76 \\
\hline $\mathrm{d} 067022$ & 77 \\
\hline $\mathrm{d} 067022$ & 78 \\
\hline $\mathrm{d} 067022$ & 81 \\
\hline $\mathrm{d} 067022$ & 82 \\
\hline $\mathrm{d} 067022$ & 83 \\
\hline $\mathrm{d} 067022$ & 84 \\
\hline & \\
\hline
\end{tabular}

Avg. Vel.

Vel. Std. Dev.

\begin{tabular}{|l|r|}
\hline 5.34 & Vel. S \\
\hline 4.83 & 0.1 \\
\hline 4.31 & 0.1 \\
\hline 4.89 & 0.0 \\
\hline
\end{tabular}

0.10
0.19
0.08
0.01

d075021

d075021

d075021

d075021

d075021

d075021

d075021

d075021

d075021

d075021

d075021

d075021

d075021

d075021

d075021

d075021

d075021

d075021

d075021

d075021

d075021

d075021

d075021

d075021

d075021

d075021

d075021

d075021

d075021

d075021
4.89

4.83

4.65

4.45

4.19

4.14

4.13

4.22

8.51

8.39

8.44

8.70

8.56

0.02

0.08

0.03

0.03

0.01

0.01

0.08

0.04

0.03

0.03

0.01

0.07

0.08

8.89

9.19

9.80

9.94

9.84

9.00

9.17

8.86

8.65

8.52

8.38

8.25

8.21

8.23

8.47

8.39

8.28

8.07

7.90

7.89

7.93

8.15

8.11

8.10

8.16

8.08

8.16

7.61
0.04

0.12

0.08

0.03

0.02

0.07

0.03

0.14

0.01

0.06

0.05

0.01

0.05

0.06

0.04

0.02

0.08

0.07

0.03

0.02

0.05

0.03

0.04

0.05

0.01

0.03

0.01

0.09
Avg. Yaw

Yaw Std. Dev.

\begin{tabular}{|l|l|}
\hline 14.26 & 1.72 \\
\hline 19.86 & 1.25 \\
\hline 15.88 & 1.30 \\
\hline-5.79 & 0.42 \\
\hline
\end{tabular}

$-5.47$

$-10.67$

$-10.61$

$-10.21$

$-7.81$

$-12.77$

$-13.68$

\begin{tabular}{l|l}
\hline 0.46 & 1.86
\end{tabular}

\begin{tabular}{l|l}
-1.85 & 1.16
\end{tabular}

$-2.96$

2.65

\begin{tabular}{l|l}
2.65 & 0.48 \\
\hline & 1.44
\end{tabular}

\begin{tabular}{l|l}
6.53 & 1.85 \\
\hline 0.93
\end{tabular}

\begin{tabular}{l|l}
0.93 & 2.38
\end{tabular}

\begin{tabular}{l|l}
0.93 & 2.02 \\
\hline-3.89
\end{tabular}

\begin{tabular}{l|l}
-0.02 & 1.40 \\
-5.41 & 1.96
\end{tabular}

$-5.41$

$-5.13$

$-5.56$

$-6.67$

$-6.16$

$-4.00$

$-5.16$

$-6.02$

$-7.10$

$-8.12$

$-4.35$

$-0.69$

2.78

\begin{tabular}{l|l}
1.51 & 0.62 \\
\hline 3.79
\end{tabular}

3.79

2.93

1.80

2.10

0.01

$-0.12$

4.49

0.76

0.47

1.29

0.75

0.75

1.19

4.82

2.31

2.60

2.07

0.49

1.42

1.23

0.48

1.44

1.38

3.01

2.75

1.39

0.91

0.81

0.58

1.71

0.78

0.72

0.76

2.74

1.11

1.35

1.10

0.48

0.49

0.57

1.45

0.44

2.04

2.41

2.41
0.43
1.03

1.03
2.51




\begin{tabular}{|c|c|c|c|c|c|}
\hline Tape & Cycle & Avg. Vel. & Vel. Std. Dev. & Avg. Yaw & Yaw Std. Dev. \\
\hline $\mathrm{d} 067031$ & 210 & 11.45 & 0.03 & -1.62 & 1.89 \\
\hline $\mathrm{d} 067031$ & 211 & 11.33 & 0.12 & -1.43 & 0.78 \\
\hline d067031 & 213 & 11.24 & 0.10 & -0.75 & 3.34 \\
\hline $\mathrm{d} 067031$ & 216 & 10.53 & $\overline{0.09}$ & 1.32 & 2.15 \\
\hline $\mathrm{d} 067031$ & 217 & 10.76 & 0.13 & 0.32 & 1.99 \\
\hline d067031 & 219 & 10.63 & 0.12 & -1.35 & 1.06 \\
\hline $\mathrm{d} 067031$ & 220 & 10.49 & 0.06 & -9.86 & 2.77 \\
\hline $\mathrm{d} 067031$ & 221 & 10.18 & 0.12 & -4.10 & 1.74 \\
\hline d067031 & 226 & 9.45 & 0.17 & 9.35 & 3.77 \\
\hline $\mathrm{d} 067031$ & 229 & 9.67 & 0.24 & -0.01 & 0.82 \\
\hline $\mathrm{d} 067031$ & 231 & $\overline{9.36}$ & 0.02 & 0.75 & 3.57 \\
\hline $\mathrm{d} 067031$ & 242 & 10.31 & 0.05 & 2.08 & 0.57 \\
\hline d067031 & 246 & 10.54 & 0.12 & 5.34 & 4.25 \\
\hline $\mathrm{d} 067031$ & 247 & 9.99 & 0.11 & 3.03 & 2.79 \\
\hline $\mathrm{d} 067031$ & 248 & 9.86 & 0.05 & 3.42 & 5.19 \\
\hline d067031 & 249 & 9.47 & 0.10 & -0.25 & 7.29 \\
\hline $\mathrm{d} 067031$ & 254 & 8.96 & 0.06 & -6.99 & 3.28 \\
\hline $\mathrm{d} 073011$ & 63 & 16.02 & 0.07 & 3.62 & 2.44 \\
\hline $\mathrm{d} 073011$ & 69 & 15.26 & 0.14 & 1.88 & 0.87 \\
\hline $\mathrm{d} 071021$ & 311 & 17.93 & 0.11 & 10.05 & 1.24 \\
\hline d071021 & 312 & 17.25 & 0.16 & 5.87 & 3.17 \\
\hline $\mathrm{d} 071021$ & 313 & 16.45 & 0.35 & 0.16 & 5.29 \\
\hline $\mathrm{d} 071021$ & 314 & 15.14 & 0.25 & -7.93 & 0.98 \\
\hline $\mathrm{d} 071021$ & 315 & 14.52 & 0.15 & -14.39 & 7.36 \\
\hline $\mathrm{d} 071021$ & 317 & 15.86 & 0.23 & -32.01 & 4.43 \\
\hline $\mathrm{d} 071021$ & 320 & 15.82 & 0.08 & -2.48 & 5.74 \\
\hline $\mathrm{d} 071021$ & 321 & 16.98 & 0.63 & 6.41 & 4.51 \\
\hline $\mathrm{d} 071021$ & 324 & 23.02 & 0.14 & 13.11 & 4.42 \\
\hline $\mathrm{d} 071021$ & 325 & 21.65 & 1.02 & 8.20 & 1.52 \\
\hline $\mathrm{d} 071021$ & 326 & 18.98 & 0.32 & -2.85 & 6.75 \\
\hline $\mathrm{d} 071021$ & 327 & 18.94 & 0.08 & -16.08 & 1.49 \\
\hline $\mathrm{d} 071021$ & 329 & 23.12 & 0.52 & -18.45 & 1.37 \\
\hline $\mathrm{d} 071021$ & 337 & 20.59 & $\overline{0.66}$ & -0.04 & 3.51 \\
\hline $\mathrm{d} 071021$ & 338 & 19.71 & 0.44 & -1.21 & 3.44 \\
\hline $\mathrm{d} 071021$ & 339 & 19.00 & 0.49 & -9.72 & 3.70 \\
\hline $\mathrm{d} 071021$ & 350 & 23.24 & 0.34 & 4.33 & 0.78 \\
\hline $\mathrm{d} 071021$ & 352 & 21.58 & 0.41 & 0.83 & 1.95 \\
\hline $\mathrm{d} 071021$ & 353 & 19.86 & 0.55 & 1.44 & 1.88 \\
\hline
\end{tabular}


CYCLE-AVERAGED INFLOW AND STANDARD DEVIATION VALUES AT 80\% SPAN FOR THE HIGHLY CORRELATED CYCLES $(>0.8)$ FROM THE 50-CYCLE SETS.

Note: The $80 \%$ span data could not be used for tape d073011 due to errors in the data.

\begin{tabular}{|c|c|c|c|c|c|}
\hline Tape & Cycle & Avg. Vel. & Vel. Std. Dev. & Avg. Yaw & Yaw Std. Dev. \\
\hline d067022 & 37 & 5.80 & 0.10 & 10.63 & 0.50 \\
\hline $\mathrm{d} 067022$ & 38 & 5.95 & 0.04 & 11.53 & 0.93 \\
\hline $\mathrm{d} 067022$ & 39 & 5.78 & 0.04 & 12.98 & 0.63 \\
\hline $\mathrm{d} 067022$ & 40 & 5.56 & 0.10 & 11.15 & 3.54 \\
\hline $\mathrm{d} 067022$ & 41 & 5.21 & 0.08 & 4.12 & 0.29 \\
\hline $\mathrm{d} 067022$ & 42 & 4.98 & 0.02 & 2.44 & 1.29 \\
\hline d067022 & $\overline{43}$ & 4.99 & 0.03 & -3.01 & 3.05 \\
\hline $\mathrm{d} 067022$ & 44 & 5.15 & 0.04 & -8.87 & 0.38 \\
\hline $\mathrm{d} 067022$ & 45 & 5.37 & 0.05 & -7.73 & 2.12 \\
\hline d067022 & 46 & 5.43 & 0.00 & -13.76 & 1.96 \\
\hline $\mathrm{d} 067022$ & 48 & 5.57 & 0.01 & -2.06 & 0.48 \\
\hline $\mathrm{d} 067022$ & 52 & 5.91 & 0.03 & -1.58 & 1.55 \\
\hline $\mathrm{d} 067022$ & 53 & 6.02 & 0.04 & 2.20 & 1.73 \\
\hline $\mathrm{d} 067022$ & 54 & 6.10 & 0.02 & 6.64 & 0.93 \\
\hline $\mathrm{d} 067022$ & 55 & 5.94 & 0.07 & 5.30 & 2.02 \\
\hline d067022 & 56 & 5.75 & 0.04 & 4.99 & 1.40 \\
\hline $\mathrm{d} 067022$ & 57 & 5.67 & 0.06 & 0.26 & 2.58 \\
\hline $\mathrm{d} 067022$ & 63 & 5.55 & 0.07 & 3.64 & 4.03 \\
\hline $\mathrm{d} 067022$ & 65 & 5.34 & 0.10 & 14.26 & 1.72 \\
\hline $\mathrm{d} 067022$ & 66 & 4.83 & 0.19 & 19.86 & 1.25 \\
\hline $\mathrm{d} 067022$ & 67 & 4.31 & 0.08 & 15.88 & 1.30 \\
\hline $\mathrm{d} 067022$ & 75 & 4.89 & 0.01 & -5.79 & 0.42 \\
\hline $\mathrm{d} 067022$ & 76 & 4.83 & 0.02 & $-5 . \overline{47}$ & 1.19 \\
\hline $\mathrm{d} 067022$ & 77 & 4.65 & 0.08 & -10.67 & 4.82 \\
\hline $\mathrm{d} 067022$ & 78 & 4.45 & 0.03 & -10.61 & 2.31 \\
\hline $\mathrm{d} 067022$ & 81 & 4.19 & 0.03 & -10.21 & 2.60 \\
\hline $\mathrm{d} 067022$ & 83 & 4.13 & 0.01 & -12.77 & 0.49 \\
\hline d067022 & 84 & 4.22 & 0.08 & -13.68 & 1.42 \\
\hline & & & & & \\
\hline $\mathrm{d} 075021$ & 5 & 8.67 & 0.03 & -3.05 & 0.35 \\
\hline $\mathrm{d} 075021$ & 6 & 8.51 & 0.04 & 0.46 & 1.86 \\
\hline $\mathrm{d} 075021$ & 7 & 8.39 & 0.03 & -1.85 & 1.16 \\
\hline $\mathrm{d} 075021$ & 8 & 8.44 & 0.03 & -2.96 & 1.23 \\
\hline $\mathrm{d} 075021$ & 9 & 8.54 & 0.08 & -2.29 & 1.28 \\
\hline $\mathrm{d} 075021$ & 10 & 8.78 & 0.06 & -2.96 & 1.66 \\
\hline $\mathrm{d} 075021$ & 11 & 8.46 & 0.14 & 0.45 & 2.61 \\
\hline $\mathrm{d} 075021$ & 12 & 8.01 & 0.13 & 8.66 & 3.16 \\
\hline $\mathrm{d} 075021$ & 16 & 8.70 & 0.01 & 2.65 & 0.48 \\
\hline $\mathrm{d} 075021$ & 17 & 8.56 & 0.07 & 4.09 & 1.44 \\
\hline $\mathrm{d} 075021$ & 18 & 8.78 & 0.09 & 5.25 & 1.73 \\
\hline $\mathrm{d} 075021$ & 19 & 9.04 & 0.08 & 6.53 & 1.85 \\
\hline $\mathrm{d} 075021$ & 20 & 9.00 & 0.08 & 6.43 & 1.43 \\
\hline $\mathrm{d} 075021$ & 21 & 8.90 & 0.05 & 0.77 & 0.80 \\
\hline
\end{tabular}


Tape

Cycle

Avg. Vel.

Vel. Std. Dev.

Avg. Yaw Yaw Std. Dev.

\begin{tabular}{|c|c|c|c|c|c|}
\hline $\mathrm{d} 075021$ & 22 & 8.89 & 0.04 & 0.93 & 2.38 \\
\hline $\mathrm{d} 075021$ & 23 & 9.19 & 0.12 & -0.02 & 1.40 \\
\hline $\mathrm{d} 075021$ & 24 & 9.55 & 0.10 & 2.64 & 2.31 \\
\hline $\mathrm{d} 075021$ & 25 & 9.80 & 0.08 & -3.89 & 1.96 \\
\hline $\mathrm{d} 075021$ & 26 & 9.94 & 0.03 & -5.41 & 3.01 \\
\hline $\mathrm{d} 075021$ & 27 & 9.84 & 0.02 & -5.13 & 2.75 \\
\hline $\mathrm{d} 075021$ & 29 & 9.11 & 0.11 & -4.18 & 0.62 \\
\hline $\mathrm{d} 075021$ & 30 & 9.00 & 0.07 & -5.56 & 1.39 \\
\hline $\mathrm{d} 075021$ & 31 & 9.17 & 0.03 & -6.67 & 0.91 \\
\hline $\mathrm{d} 075021$ & 32 & 8.86 & 0.14 & -6.16 & 0.81 \\
\hline $\mathrm{d} 075021$ & 33 & 8.65 & 0.01 & -4.00 & 0.58 \\
\hline $\mathrm{d} 075021$ & 34 & 8.52 & $\overline{0.06}$ & -5.16 & 1.71 \\
\hline $\mathrm{d} 075021$ & 35 & 8.38 & 0.05 & -6.02 & 0.78 \\
\hline $\mathrm{d} 075021$ & 36 & 8.25 & 0.01 & -7.10 & 0.72 \\
\hline $\mathrm{d} 075021$ & 37 & 8.21 & 0.05 & -8.12 & 0.76 \\
\hline $\mathrm{d} 075021$ & 38 & 8.23 & 0.06 & -4.35 & 2.74 \\
\hline $\mathrm{d} 075021$ & 39 & 8.47 & 0.04 & -0.69 & 1.11 \\
\hline $\mathrm{d} 075021$ & 44 & 7.89 & 0.02 & 1.80 & 0.49 \\
\hline $\mathrm{d} 075021$ & 45 & 7.93 & 0.05 & 2.10 & 0.57 \\
\hline $\mathrm{d} 075021$ & 46 & 8.15 & 0.03 & 0.01 & 1.45 \\
\hline $\mathrm{d} 075021$ & 47 & 8.11 & 0.04 & -0.12 & $\overline{0.44}$ \\
\hline $\mathrm{d} 075021$ & $\overline{48}$ & 8.10 & 0.05 & 4.49 & 2.04 \\
\hline $\mathrm{d} 075021$ & $\overline{49}$ & $\overline{8.16}$ & 0.01 & 0.76 & 2.41 \\
\hline $\mathrm{d} 075021$ & 50 & $\overline{8.08}$ & 0.03 & 0.47 & $\overline{0.43}$ \\
\hline $\mathrm{d} 075021$ & 51 & 8.16 & 0.01 & 1.29 & 1.03 \\
\hline $\mathrm{d} 075021$ & 52 & 7.97 & 0.11 & 0.78 & 2.56 \\
\hline $\mathrm{d} 075021$ & 53 & 7.61 & 0.09 & 0.75 & 2.51 \\
\hline $\mathrm{d} 075021$ & 54 & 7.46 & 0.03 & -3.01 & 1.89 \\
\hline $\mathrm{d} 067031$ & 210 & 11.45 & 0.03 & -1.62 & 1.89 \\
\hline $\mathrm{d} 067031$ & 211 & 11.33 & 0.12 & -1.43 & 0.78 \\
\hline $\mathrm{d} 067031$ & 212 & 11.13 & 0.02 & 1.27 & 0.87 \\
\hline $\mathrm{d} 067031$ & 213 & 11.24 & 0.10 & -0.75 & 3.34 \\
\hline $\mathrm{d} 067031$ & 216 & 10.53 & 0.09 & 1.32 & 2.15 \\
\hline $\mathrm{d} 067031$ & 217 & 10.76 & 0.13 & 0.32 & 1.99 \\
\hline $\mathrm{d} 067031$ & 218 & 10.89 & 0.03 & -1.19 & 1.04 \\
\hline $\mathrm{d} 067031$ & 219 & 10.63 & 0.12 & -1.35 & 1.06 \\
\hline $\mathrm{d} 067031$ & 220 & 10.49 & 0.06 & -9.86 & 2.77 \\
\hline $\mathrm{d} 067031$ & 221 & 10.18 & 0.12 & -4.10 & 1.74 \\
\hline $\mathrm{d} 067031$ & 223 & 10.57 & 0.04 & -1.29 & 1.32 \\
\hline $\mathrm{d} 067031$ & 234 & 9.53 & 0.02 & 6.11 & $\overline{4.64}$ \\
\hline $\mathrm{d} 067031$ & 236 & 9.98 & 0.13 & 1.06 & 1.21 \\
\hline $\mathrm{d} 067031$ & 241 & 10.44 & 0.01 & 2.43 & 0.48 \\
\hline $\mathrm{d} 067031$ & 243 & 10.24 & 0.02 & 2.40 & 0.53 \\
\hline $\mathrm{d} 067031$ & 244 & 10.34 & 0.06 & 3.25 & 0.64 \\
\hline $\mathrm{d} 067031$ & 245 & 10.62 & 0.05 & 6.00 & 0.83 \\
\hline $\mathrm{d} 067031$ & 246 & 10.54 & 0.12 & 5.34 & 4.25 \\
\hline
\end{tabular}


Tape

Cycle

Avg. Vel.

Vel. Std. Dev.

Avg. Yaw

Yaw Std. Dev.

\begin{tabular}{|c|c|c|c|c|c|}
\hline $\mathrm{d} 067031$ & 247 & 9.99 & 0.11 & 3.03 & 2.79 \\
\hline $\mathrm{d} 067031$ & 248 & 9.86 & 0.05 & 3.42 & 5.19 \\
\hline $\mathrm{d} 067031$ & 249 & 9.47 & 0.10 & -0.25 & 7.29 \\
\hline $\mathrm{d} 071021$ & 311 & 17.93 & 0.11 & 10.05 & 1.24 \\
\hline $\mathrm{d} 071021$ & 312 & 17.25 & 0.16 & 5.87 & 3.17 \\
\hline $\mathrm{d} 071021$ & 314 & 15.14 & 0.25 & -7.93 & 0.98 \\
\hline $\mathrm{d} 071021$ & 315 & 14.52 & 0.15 & -14.39 & 7.36 \\
\hline $\mathrm{d} 071021$ & 316 & 15.42 & 0.27 & -22.47 & 2.99 \\
\hline $\mathrm{d} 071021$ & 317 & 15.86 & 0.23 & -32.01 & 4.43 \\
\hline $\mathrm{d} 071021$ & 320 & 15.82 & 0.08 & -2.48 & 5.74 \\
\hline $\mathrm{d} 071021$ & 321 & 16.98 & 0.63 & 6.41 & 4.51 \\
\hline $\mathrm{d} 071021$ & 324 & 23.02 & 0.14 & 13.11 & 4.42 \\
\hline $\mathrm{d} 071021$ & 325 & 21.65 & 1.02 & 8.20 & 1.52 \\
\hline $\mathrm{d} 071021$ & 326 & 18.98 & 0.32 & -2.85 & 6.75 \\
\hline $\mathrm{d} 071021$ & 327 & 18.94 & 0.08 & -16.08 & 1.49 \\
\hline $\mathrm{d} 071021$ & 329 & 23.12 & 0.52 & -18.45 & 1.37 \\
\hline $\mathrm{d} 071021$ & 338 & 19.71 & 0.44 & -1.21 & 3.44 \\
\hline $\mathrm{d} 071021$ & 352 & 21.58 & 0.41 & 0.83 & 1.95 \\
\hline $\mathrm{d} 071021$ & 353 & 19.86 & 0.55 & 1.44 & 1.88 \\
\hline $\mathrm{d} 071021$ & 354 & 19.74 & 0.20 & 2.75 & 2.53 \\
\hline
\end{tabular}




\section{Appendix C}

CYCLE-AVERAGED INFLOW AND STANDARD DEVIATION VALUES FOR THE 50 CYCLES USED IN THE $-20^{\circ}$ YAW PERFORMANCE BASELINE.

\begin{tabular}{|c|c|c|c|c|c|}
\hline Tape & Cycle & Avg. Vel. & Vel. Std. Dev. & Avg. Yaw & Yaw Std. Dev. \\
\hline $\mathrm{d} 065012$ & 124 & 6.19 & 0.02 & -20.28 & 0.66 \\
\hline $\mathrm{d} 065022$ & 14 & 4.77 & 0.01 & -19.38 & 0.70 \\
\hline d066021 & 321 & 7.41 & 0.29 & -20.71 & 0.57 \\
\hline d067011 & 124 & 9.78 & 0.32 & -21.19 & 0.64 \\
\hline $\mathrm{d} 067022$ & 224 & 2.36 & 0.00 & -20.36 & 0.48 \\
\hline $\mathrm{d} 067022$ & 263 & 3.43 & 0.04 & -19.42 & 0.38 \\
\hline $\mathrm{d} 067031$ & 54 & 11.14 & 0.22 & -19.20 & 0.88 \\
\hline $\mathrm{d} 067031$ & 307 & 10.56 & 0.13 & -21.68 & 0.40 \\
\hline $\mathrm{d} 068022$ & 414 & 13.44 & 0.15 & -19.16 & 0.81 \\
\hline $\mathrm{d} 068022$ & 415 & 13.54 & 0.18 & -19.67 & 1.07 \\
\hline $\mathrm{d} 069031$ & 344 & 6.94 & 0.01 & -21.67 & 0.52 \\
\hline d069032 & 245 & 5.24 & 0.02 & -19.80 & 0.69 \\
\hline d069032 & 268 & $\overline{3.64}$ & 0.01 & -21.00 & 0.26 \\
\hline $\mathrm{d} 070021$ & 342 & 6.93 & 0.01 & -20.26 & 0.80 \\
\hline d070022 & 20 & 6.06 & 0.05 & -21.02 & 0.39 \\
\hline $\mathrm{d} 070031$ & 204 & 5.00 & 0.15 & -21.31 & 0.58 \\
\hline $\mathrm{d} 070031$ & 291 & 4.36 & 0.06 & -21.14 & 0.18 \\
\hline $\mathrm{d} 070032$ & 51 & 5.24 & 0.06 & -19.25 & 0.30 \\
\hline $\mathrm{d} 071011$ & 88 & 12.96 & 0.31 & -19.46 & 0.67 \\
\hline $\mathrm{d} 071012$ & 121 & 15.37 & 0.15 & -19.43 & 0.83 \\
\hline d071021. & 329 & 23.12 & 0.52 & -18.45 & 1.37 \\
\hline $\mathrm{d} 071041$ & 296 & 11.45 & 0.42 & -18.37 & $\overline{0.94}$ \\
\hline $\mathrm{d} 071042$ & 111 & 17.39 & 0.09 & -19.59 & 0.79 \\
\hline d072011 & 351 & 14.87 & 0.30 & -18.21 & 0.57 \\
\hline $\mathrm{d} 072011$ & 352 & 15.93 & 0.26 & -19.73 & 0.59 \\
\hline $\mathrm{d} 072012$ & 3 & 12.29 & 0.14 & -20.50 & 0.33 \\
\hline $\mathrm{d} 072012$ & 102 & 14.45 & 0.59 & -21.11 & 0.74 \\
\hline $\mathrm{d} 072012$ & 347 & 19.53 & 0.45 & -19.67 & 0.74 \\
\hline $\mathrm{d} 072012$ & 356 & 22.30 & 0.24 & -18.20 & 1.59 \\
\hline $\mathrm{d} 072022$ & 345 & 20.09 & 0.20 & -21.67 & 0.71 \\
\hline $\mathrm{d} 072022$ & 347 & 19.44 & 0.13 & -18.57 & 0.72 \\
\hline $\mathrm{d} 072022$ & 348 & 19.22 & 0.04 & -20.84 & 0.44 \\
\hline $\mathrm{d} 072022$ & 352 & 18.96 & 0.02 & -21.46 & 0.71 \\
\hline $\mathrm{d} 072031$ & 57 & 18.56 & 0.13 & -20.33 & 1.38 \\
\hline $\mathrm{d} 072031$ & 269 & 14.29 & 0.19 & -21.35 & 0.42 \\
\hline $\mathrm{d} 072031$ & 322 & 9.46 & 0.08 & -18.91 & 0.51 \\
\hline $\mathrm{d} 072032$ & 64 & 16.87 & 0.36 & -19.17 & 0.61 \\
\hline $\mathrm{d} 072032$ & 67 & 18.25 & 0.19 & -20.57 & 1.04 \\
\hline d072032 & 87 & 13.94 & 0.23 & -18.47 & 0.73 \\
\hline $\mathrm{d} 072041$ & 94 & 9.03 & 0.10 & -19.24 & 0.99 \\
\hline $\mathrm{d} 072041$ & 174 & 15.87 & 0.10 & -20.91 & 0.60 \\
\hline
\end{tabular}


Tape

Cycle

Avg. Vel.

Vel. Std. Dev.

Avg. Yaw

Yaw Std. Dev.

\begin{tabular}{|c|c|c|c|c|c|}
\hline $\mathrm{d} 072041$ & 191 & 15.99 & 0.32 & -19.12 & 0.88 \\
\hline $\mathrm{d} 072042$ & 42 & 9.78 & 0.08 & -19.56 & 0.61 \\
\hline $\mathrm{d} 072042$ & 198 & 8.79 & 0.32 & -18.94 & 0.94 \\
\hline $\mathrm{d} 073021$ & 193 & 10.65 & 0.09 & -20.20 & 0.61 \\
\hline $\mathrm{d} 073021$ & 222 & 11.18 & 0.12 & -20.87 & 0.79 \\
\hline $\mathrm{d} 073031$ & 163 & 9.24 & 0.03 & -19.09 & 0.87 \\
\hline $\mathrm{d} 073031$ & 165 & 8.98 & 0.05 & -21.03 & 0.66 \\
\hline $\mathrm{d} 073031$ & 168 & 8.01 & 0.02 & -18.58 & 0.28 \\
\hline $\mathrm{d} 075021$ & 259 & 8.37 & 0.07 & -19.86 & 0.29 \\
\hline
\end{tabular}

CYCLE-AVERAGED INFLOW AND STANDARD DEVIATION VALUES FOR THE 50 CYCLES USED IN THE $-10^{\circ}$ YAW PERFORMANCE BASELINE.

$\begin{array}{lllll}\text { Tape } & \text { Cycle } & \text { Avg. Vel. } & \text { Vel. Std. Dev. } & \text { Avg. Yaw }\end{array}$

\begin{tabular}{|c|c|c|c|c|c|}
\hline d065011 & 24 & 8.40 & 0.04 & -9.46 & 0.40 \\
\hline $\mathrm{d} 065022$ & 117 & 5.88 & 0.14 & -10.75 & 0.46 \\
\hline $\mathrm{d} 066021$ & 269 & 10.48 & 0.01 & -10.08 & 0.43 \\
\hline $\mathrm{d} 066022$ & 303 & 8.39 & 0.07 & -9.68 & 0.32 \\
\hline $\mathrm{d} 066031$ & 292 & 13.90 & 0.34 & -9.68 & 0.43 \\
\hline $\mathrm{d} 066032$ & 91 & 9.77 & 0.12 & -9.87 & 0.54 \\
\hline d067021 & 211 & 9.09 & 0.06 & -9.82 & 0.56 \\
\hline $\mathrm{d} 067022$ & 96 & 5.98 & 0.05 & -9.50 & 0.46 \\
\hline $\mathrm{d} 067022$ & 132 & 4.65 & 0.02 & -10.53 & 0.44 \\
\hline $\mathrm{d} 067022$ & 237 & 3.04 & 0.02 & -10.06 & 0.33 \\
\hline $\mathrm{d} 067032$ & 324 & 14.13 & 0.10 & -10.95 & 0.32 \\
\hline d068011 & 231 & 12.74 & 0.19 & -10.56 & 0.39 \\
\hline $\mathrm{d} 068012$ & 160 & 17.00 & 0.12 & -10.97 & 0.61 \\
\hline $\mathrm{d} 068021$ & 287 & 16.29 & 0.25 & -9.09 & 0.23 \\
\hline $\mathrm{d} 068021$ & 301 & 17.54 & 0.06 & -9.52 & 0.37 \\
\hline $\mathrm{d} 069022$ & 173 & 10.08 & 0.02 & -10.58 & 0.47 \\
\hline $\mathrm{d} 069031$ & 29 & 8.07 & 0.05 & -10.16 & 0.52 \\
\hline $\mathrm{d} 069031$ & 30 & 8.04 & 0.10 & -10.42 & 0.39 \\
\hline $\mathrm{d} 070021$ & 229 & 7.80 & 0.04 & -10.46 & 0.29 \\
\hline $\mathrm{d} 070022$ & 36 & 6.40 & 0.06 & -10.05 & 0.43 \\
\hline $\mathrm{d} 071011$ & 42 & 17.34 & 0.33 & -9.74 & 0.74 \\
\hline $\mathrm{d} 071011$ & 77 & 16.86 & 0.11 & -9.54 & 0.55 \\
\hline $\mathrm{d} 071011$ & 78 & 16.35 & 0.09 & -9.15 & 0.17 \\
\hline $\mathrm{d} 071021$ & 271 & 21.37 & 0.11 & -10.45 & 0.55 \\
\hline $\mathrm{d} 071031$ & 145 & 15.35 & 0.18 & -10.01 & 0.71 \\
\hline $\mathrm{d} 071032$ & 8 & 12.47 & 0.22 & -10.02 & 0.42 \\
\hline $\mathrm{d} 071032$ & 182 & 22.19 & 0.13 & -10.41 & 0.35 \\
\hline $\mathrm{d} 071032$ & 214 & 18.72 & 0.41 & -9.04 & 0.98 \\
\hline $\mathrm{d} 071042$ & 180 & 18.53 & 0.58 & -9.24 & 0.49 \\
\hline $\mathrm{d} 071042$ & 190 & 20.22 & 0.45 & -10.31 & 0.81 \\
\hline $\mathrm{d} 072012$ & 87 & 11.88 & 0.17 & -9.77 & 0.51 \\
\hline $\mathrm{d} 072012$ & 149 & 11.87 & 0.16 & -10.40 & 0.40 \\
\hline $\mathrm{d} 072012$ & 334 & 15.20 & 0.11 & -9.49 & 0.59 \\
\hline
\end{tabular}




\begin{tabular}{|c|c|c|c|c|c|}
\hline Tape & Cycle & Avg. Vel. & Vel. Std. Dev. & Avg. Yaw & Yaw Std. Dev. \\
\hline $\mathrm{d} 072021$ & 157 & 20.45 & 0.15 & -10.49 & 0.98 \\
\hline d072041 & 292 & 9.50 & 0.09 & -9.65 & 0.52 \\
\hline d072042 & 62 & 8.10 & 0.05 & -10.03 & 0.34 \\
\hline d072042 & 72 & 6.93 & 0.02 & -10.36 & 0.30 \\
\hline $\mathrm{d} 073011$ & 29 & 18.12 & 0.08 & -10.43 & 0.72 \\
\hline d073011 & 131 & $\overline{14.56}$ & 0.05 & -10.51 & 0.83 \\
\hline d073012 & 64 & 9.73 & 0.05 & -10.36 & 0.55 \\
\hline $\mathrm{d} 073021$ & 2 & 10.91 & 0.04 & -10.11 & 0.49 \\
\hline d073021 & 12 & 11.21 & 0.02 & -9.17 & 0.44 \\
\hline $\mathrm{d} 073021$ & 119 & 11.86 & 0.37 & -9.78 & 0.55 \\
\hline $\mathrm{d} 073022$ & 63 & 5.63 & 0.02 & -9.45 & 0.68 \\
\hline d073032 & 141 & 7.17 & 0.01 & -10.45 & 0.45 \\
\hline $\mathrm{d} 073032$ & 142 & 7.11 & 0.02 & -9.75 & 0.34 \\
\hline d073041 & 24 & 3.85 & 0.04 & -10.25 & 0.87 \\
\hline $\mathrm{d} 075011$ & 139 & 10.41 & 0.14 & -10.57 & 0.40 \\
\hline $\mathrm{d} 075012$ & 248 & 7.67 & 0.03 & -10.83 & 0.58 \\
\hline $\mathrm{d} 075022$ & 56 & 4.16 & 0.06 & -9.55 & 0.43 \\
\hline
\end{tabular}

CYCLE-AVERAGED INFLOW AND STANDARD DEVIATION VALUES FOR THE 50 CYCLES USED IN THE $10^{\circ}$ YAW PERFORMANCE BASELINE.

Tape

\begin{tabular}{|c|c|}
\hline $\mathrm{d} 065011$ & 140 \\
\hline $\mathrm{d} 065021$ & 126 \\
\hline $\mathrm{d} 065021$ & 213 \\
\hline $\mathrm{d} 065022$ & 190 \\
\hline $\mathrm{d} 066032$ & 18 \\
\hline $\mathrm{d} 066032$ & 32 \\
\hline $\mathrm{d} 067021$ & 185 \\
\hline $\mathrm{d} 067022$ & 37 \\
\hline $\mathrm{d} 067022$ & 179 \\
\hline $\mathrm{d} 068021$ & 205 \\
\hline $\mathrm{d} 068022$ & 111 \\
\hline $\mathrm{d} 070021$ & 155 \\
\hline $\mathrm{d} 070021$ & 221 \\
\hline $\mathrm{d} 070022$ & 142 \\
\hline $\mathrm{d} 070031$ & 158 \\
\hline $\mathrm{d} 070032$ & 318 \\
\hline $\mathrm{d} 070032$ & 345 \\
\hline $\mathrm{d} 070041$ & 57 \\
\hline $\mathrm{d} 071021$ & 311 \\
\hline $\mathrm{d} 071031$ & 74 \\
\hline $\mathrm{d} 071031$ & 106 \\
\hline $\mathrm{d} 071032$ & 97 \\
\hline $\mathrm{d} 071032$ & 308 \\
\hline $\mathrm{d} 071041$ & 159 \\
\hline $\mathrm{d} 072012$ & 180 \\
\hline & \\
\hline
\end{tabular}

Avg. Vel.

Cycle

40

126

213

190

111

155

158

318

57

106

180

10.99

7.28

7.33

4.35

11.03

11.96

8.87

5.80

3.59

16.37

15.82

8.80

7.85

7.48

6.66

9.77

6.71

6.51

17.93

18.11

19.79

19.88

13.07

18.21

9.02

Vel. Std. Dev.

Avg. Yaw

Yaw Std. Dev.

\begin{tabular}{|c|c|c|}
\hline 0.27 & 9.60 & 1.19 \\
\hline 0.02 & 9.88 & 0.61 \\
\hline 0.04 & 10.64 & 0.64 \\
\hline 0.05 & 9.00 & 0.53 \\
\hline 0.06 & 9.04 & 0.84 \\
\hline 0.06 & 9.65 & 1.49 \\
\hline 0.08 & 10.93 & 0.56 \\
\hline 0.10 & 10.63 & 0.50 \\
\hline 0.01 & 10.77 & 0.19 \\
\hline 0.05 & 10.56 & 0.92 \\
\hline 0.08 & 9.72 & 0.79 \\
\hline 0.04 & 10.43 & 0.48 \\
\hline 0.11 & 10.16 & 0.62 \\
\hline 0.12 & 9.50 & 0.47 \\
\hline 0.07 & 10.49 & 0.24 \\
\hline 0.05 & 10.73 & 0.59 \\
\hline 0.02 & 9.91 & 0.64 \\
\hline 0.03 & 9.53 & 0.50 \\
\hline 0.11 & 10.05 & 1.24 \\
\hline 0.34 & 10.03 & 0.93 \\
\hline 0.36 & 10.87 & 1.40 \\
\hline 0.51 & 9.14 & 0.84 \\
\hline 0.23 & 9.67 & 0.93 \\
\hline 0.49 & 9.37 & 0.95 \\
\hline 0.09 & 9.64 & 0.44 \\
\hline
\end{tabular}




\begin{tabular}{|c|c|c|c|c|c|}
\hline Tape & Cycle & Avg. Vel. & Vel. Std. Dev. & Avg. Yaw & Yaw Std. Dev. \\
\hline d072012 & 224 & 19.04 & 0.30 & 9.07 & 1.23 \\
\hline d072021 & 42 & 15.34 & 0.13 & 9.99 & 1.45 \\
\hline d072021 & 44 & 14.02 & 0.25 & 10.59 & 1.32 \\
\hline d072021 & 53 & 14.11 & 0.06 & 9.46 & 1.23 \\
\hline d072021 & 270 & 12.46 & 0.20 & 9.84 & 0.58 \\
\hline d072021 & 274 & 12.15 & 0.07 & 10.50 & 0.83 \\
\hline d072022 & 87 & 21.77 & 0.14 & 9.33 & 1.09 \\
\hline d072022 & 93 & 20.96 & 0.69 & 10.65 & 1.27 \\
\hline d072031 & 85 & 16.83 & 0.09 & 10.96 & 0.69 \\
\hline d072031 & 136 & 14.10 & 0.03 & 9.18 & 0.86 \\
\hline d073011 & 182 & 13.63 & 0.12 & 9.84 & 0.68 \\
\hline d073012 & 240 & 10.44 & 0.08 & 9.97 & 1.40 \\
\hline d073022 & 235 & 9.73 & 0.02 & 10.32 & 0.55 \\
\hline d073022 & 297 & 5.55 & 0.08 & 10.48 & 0.58 \\
\hline d073032 & 112 & 5.35 & 0.03 & 10.03 & 0.23 \\
\hline d073032 & 114 & 5.28 & 0.03 & 9.33 & 0.38 \\
\hline d073032 & 115 & 5.16 & 0.06 & 10.79 & 0.56 \\
\hline d073041 & 53 & 6.81 & 0.03 & 9.98 & 0.37 \\
\hline d073042 & 25 & 10.05 & 0.07 & 10.81 & 0.42 \\
\hline d073042 & 26 & 10.11 & 0.03 & 10.62 & 0.97 \\
\hline d073042 & 158 & 4.25 & 0.01 & 9.00 & 0.55 \\
\hline d075011 & 18 & 8.85 & 0.10 & 9.34 & 0.37 \\
\hline d075011 & 76 & 8.23 & 0.08 & 9.67 & 0.29 \\
\hline d075012 & 194 & 7.09 & 0.11 & 10.41 & 0.44 \\
\hline d075021 & 143 & 8.39 & 0.03 & 10.46 & 0.16 \\
\hline
\end{tabular}

CYCLE-AVERAGED INFLOW AND STANDARD DEVIATION VALUES FOR THE 50 CYCLES USED IN THE $20^{\circ}$ YAW PERFORMANCE BASELINE.

\begin{tabular}{|c|c|c|c|c|c|}
\hline Tape & Cycle & Avg. Vel. & Vel. Std. Dev. & Avg. Yaw & Yaw Std. Dev. \\
\hline $\mathrm{d} 065011$ & 111 & 6.99 & 0.06 & 18.67 & 0.86 \\
\hline $\mathrm{d} 065012$ & 78 & 5.34 & 0.04 & 21.37 & 0.37 \\
\hline $\mathrm{d} 066022$ & 62 & 10.32 & 0.11 & 18.65 & 1.92 \\
\hline $\mathrm{d} 066031$ & 352 & 10.87 & 0.43 & 20.66 & 1.06 \\
\hline $\mathrm{d} 067012$ & 295 & 8.95 & $\overline{0.06}$ & 21.91 & 0.91 \\
\hline $\mathrm{d} 067012$ & 298 & 8.95 & 0.06 & 21.78 & 0.65 \\
\hline $\mathrm{d} 067022$ & 190 & 3.31 & 0.01 & 19.52 & 0.48 \\
\hline d067022 & 191 & 3.31 & 0.01 & 18.34 & 0.60 \\
\hline $\mathrm{d} 068012$ & 109 & $\overline{17.48}$ & 0.31 & $\overline{19.53}$ & 1.35 \\
\hline $\mathrm{d} 068021$ & 60 & 16.39 & 0.05 & 20.89 & 2.01 \\
\hline $\mathrm{d} 068021$ & 155 & 12.33 & 0.05 & 18.97 & 0.98 \\
\hline $\mathrm{d} 068022$ & 23 & 11.72 & 0.09 & 19.46 & 1.51 \\
\hline $\mathrm{d} 068022$ & 334 & 16.10 & 0.18 & 19.15 & 2.41 \\
\hline $\mathrm{d} 069022$ & 140 & 11.62 & 0.08 & 19.50 & 1.14 \\
\hline $\mathrm{d} 070011$ & 183 & 7.15 & 0.03 & 21.46 & 0.70 \\
\hline $\mathrm{d} 070022$ & 85 & 6.35 & $\overline{0.04}$ & 20.05 & 0.92 \\
\hline $\mathrm{d} 070032$ & 20 & 5.41 & 0.02 & 19.71 & 0.50 \\
\hline
\end{tabular}


Tape

Cycle

Avg. Vel.

Vel. Std. Dev.

Avg. Yaw

Yaw Std. Dev.

\begin{tabular}{|c|c|c|c|c|c|}
\hline d070041 & 109 & 6.18 & 0.05 & 18.38 & 0.87 \\
\hline $\mathrm{d} 070041$ & 110 & 5.93 & 0.08 & 19.21 & 0.55 \\
\hline $\mathrm{d070041}$ & 339 & 6.87 & 0.03 & 19.58 & 0.82 \\
\hline $\mathrm{d070042}$ & 2 & 6.74 & 0.07 & 21.51 & 0.54 \\
\hline $\mathrm{d} 070042$ & 10 & 6.81 & 0.05 & 20.14 & 0.92 \\
\hline d070042 & 283 & 6.42 & 0.02 & 18.22 & 0.45 \\
\hline $\mathrm{d} 071021$ & 54 & 15.94 & 0.14 & 18.29 & 1.93 \\
\hline $\mathrm{d} 071021$ & 117 & 22.63 & 0.20 & 18.31 & 0.76 \\
\hline $\mathrm{d} 071032$ & 304 & 12.35 & 0.20 & 20.89 & 0.53 \\
\hline $\mathrm{d} 071041$ & 185 & 17.18 & 0.54 & 19.75 & 1.71 \\
\hline d071041 & 210 & 22.75 & 0.39 & 21.00 & 2.03 \\
\hline d072011 & 106 & 7.90 & 0.18 & 19.51 & 0.71 \\
\hline d072012 & 314 & 14.15 & 0.07 & 19.91 & 2.10 \\
\hline d072021 & 57 & 13.46 & 0.16 & 20.39 & 1.85 \\
\hline $\mathrm{d} 072021$ & 289 & 12.76 & 0.18 & 18.11 & 0.84 \\
\hline d072022 & 213 & 19.27 & 0.41 & 18.39 & 0.78 \\
\hline $\mathrm{d} 072022$ & 217 & 19.53 & 0.29 & 19.56 & 2.32 \\
\hline $\mathrm{d} 072022$ & 221 & 18.49 & 0.11 & 21.93 & 0.75 \\
\hline $\mathrm{d} 072022$ & 224 & 21.64 & 0.39 & 20.59 & 1.82 \\
\hline $\mathrm{d} 072022$ & 239 & 20.77 & 0.08 & 18.02 & 1.54 \\
\hline $\mathrm{d} 072031$ & 99 & 14.49 & 0.28 & 21.92 & 1.46 \\
\hline $\mathrm{d} 072031$ & 126 & 15.22 & 0.12 & 21.04 & 1.41 \\
\hline d072031 & 207 & 13.82 & 0.14 & 20.63 & 1.70 \\
\hline $\mathrm{d} 072042$ & 346 & 10.60 & 0.36 & 19.88 & 1.18 \\
\hline $\mathrm{d} 073031$ & 188 & 11.07 & 0.05 & 18.53 & 0.90 \\
\hline $\mathrm{d} 073031$ & 305 & 7.95 & 0.05 & 20.09 & 0.41 \\
\hline $\mathrm{d} 073032$ & 212 & 4.92 & 0.01 & 21.86 & 0.53 \\
\hline $\mathrm{d} 073041$ & 140 & 5.91 & 0.07 & 20.37 & 0.87 \\
\hline $\mathrm{d} 073041$ & 270 & 5.10 & 0.01 & 20.04 & 0.57 \\
\hline $\mathrm{d} 073041$ & 287 & 4.21 & 0.06 & 19.46 & 0.39 \\
\hline $\mathrm{d} 075011$ & 3 & 10.01 & 0.10 & 18.45 & 0.74 \\
\hline $\mathrm{d} 075011$ & 7 & 9.35 & 0.07 & 19.21 & 0.16 \\
\hline $\mathrm{d} 075011$ & 10 & 9.37 & 0.09 & 21.07 & 0.42 \\
\hline
\end{tabular}


Public reporting bunden for this collection of information is estimated to average 1 hour per response, including the time for reviewing instructions, searching existing data sources, gathering and maintaining the data needed, and completing and revlewing the collection of information. Send comments regarding this burden estimate or any other aspect of this collection of information, inctuding suggestions tor reducing this burden. to Washington Headiduarters Services, Directorate for Information Operations and Reports, 1215 Jefferson Davis Hiqhway. Suite 1204, Arlington, VA 22202-4302, and to the Office of Management and Budget, Paperwork Reduction Project (0704-0188), Washington, DC 20503.
1.
2. REPORT DATE July 1995
3. REPORT TYPE AND DATES COVERED Subcontract Report

\section{TITLE AND SUBTITLE}

The Baseline Data Sets for Phase ll of the Combined Experiment

\section{6. $A U T H O R(S)$}

Mark S. Miller, Derek E. Shipley, Teresa S. Young, Michael C. Robinson, Marvin W. Luttges, David A. Simms

7. PERFORMING ORGANIZATION NAME(S) AND ADDRESS(ES)

University of Colorado, Boulder, Colorado

Campus Box 19

Boulder, Colorado 80309

National Renewable Energy Laboratory, Golden, Colorado

9. SPONSORINGMONITORING AGENCY NAME(S) AND ADDRESS(ES)

National Renewable Energy Laboratory

1617 Cole Blvd.

Golden, CO $80401-3393$
5. FUNDING NUMBERS

C: XAO-2-12236-01-103983

TA: WE518110

8. PERFORMING ORGANIZATION REPORT NUMBER
10. SPONSORINGMONITORING AGENCY REPORT NUMBER

TP-442-6915

DE95009271

11. SUPPLEMENTARY NOTES

NREL Technical Monitor: David A. Simms

12a. DISTRIBUTION/AVAILABILITY STATEMENT National Technical Information Service

U.S. Department of Commerce

5285 Port Royal Road

Springfield, VA 22161

12b. DISTRIBUTION CODE

UC-1211

13. ABSTRACT (Maximum 200 words)

The National Renewable Energy Laboratory's "Combined Experiment" was initiated to provide an understanding of horizontal axis wind turbine aerodynamics and their effect on the turbine structure. To this end, aerodynamic and structural baseline data sets have been defined that examine turbine performance under certain conditions. These baseline results have been discussed thoroughly in several papers. This report is an addendum to those papers and supplies additional information about the data selected in creating the baseline data sets. Several appendices are included which contain the tape and cycle numbers of the selected data, along with the average and standard deviation values for the inflow conditions, velocity, and yaw.

14. SUBJECT TERMS

horizontal axis wind turbine aerodynamics
15. NUMBER OF PAGES

16. PRICE CODE

20. LIMITATION OF ABSTRACT

UL

Standard Form 298 (Rev. 2-89) Prescribed by ANSI Sid. Z39-18
18. SECURITY CLASSIFICATION

Unclassified
19. SECURITY CLASSIFICATION OF ABSTRACT Unclassified 